\title{
Characterization of Critical Determinants of ACE2-RBD Interaction
}

4 Emily E. F. Brown ${ }^{1,2 \dagger}$, Reza Rezaei ${ }^{1,2 \dagger}$, Taylor Jamieson ${ }^{1,2 \dagger}$, Jaahnavi Dave ${ }^{1,2 \dagger}$, Nikolas T.

5 Martin $^{1,2}$, Ragunath Singaravelu ${ }^{1,2}$, Mathieu J.F. Crupi ${ }^{1,2}$, Stephen Boulton ${ }^{1,2}$, Sarah Tucker ${ }^{1,2}$,

6 Jessie Duong ${ }^{1,2}$, Joanna Poutou ${ }^{1,2}$, Adrian Pelin ${ }^{1,2}$, Hamed Yasavoli-Sharahi ${ }^{4}$, Zaid Taha ${ }^{1,2}$,

7 Rozanne Arulanandam ${ }^{1,2}$, Abera Surendran $^{1,2}$, Mina Ghahremani ${ }^{3}$, Bradley Austin ${ }^{1,2}$, Chantal

8 Matar $^{4}$, Jean-Simon Diallo ${ }^{1,2}$, John C. Bell ${ }^{1,2}$, Carolina S. Ilkow ${ }^{1,2}$, and Taha Azad ${ }^{1,2 *}$

$9{ }^{1}$ Ottawa Hospital Research Institute, Ottawa, ON, K1H 8L6, Canada.

$10{ }^{2}$ Department of Biochemistry, Microbiology and Immunology, University of Ottawa, Ottawa,

11 ON K1H 8M5, Canada.

$12{ }^{3}$ Department of Biology, University of Ottawa, Ottawa, ON, K1N 6N5, Canada.

$13{ }^{4}$ Cellular and Molecular Medicine Department, Faculty of Medicine, University of Ottawa,

14 Canada

$15 *$ Correspondence: tazad@ohri.ca; Tel.: +1-613-737-8899 (ext. 75208).

$16 \dagger$ These authors contributed equally. 
Despite sequence similarity to SARS-CoV-1, SARS-CoV-2 has demonstrated greater

24 widespread virulence and unique challenges to researchers aiming to study its pathogenicity in

25 humans. The interaction of the viral receptor binding domain (RBD) with its main host cell

26 receptor, angiotensin-converting enzyme 2 (ACE2), has emerged as a critical focal point for the

27 development of anti-viral therapeutics and vaccines. Utilizing our recently developed NanoBiT

28 technology-based biosensor, we selectively identify and characterize the impact of mutating

29 certain amino acid residues in the RBD of SARS-CoV-2 and in ACE2. Specifically, we examine

30 the mutational effects on RBD-ACE2 binding ability, before and after the addition of competitive

31 inhibitors, as well as neutralizing antibody activity. These critical determinants of virus-host

32 interactions may provide more effective targets for ongoing vaccines, drug development, and

33 potentially pave the way for determining the genetic variation underlying disease severity.

34 Keywords: SARS-CoV-2; angiotensin-converting enzyme 2; receptor binding domain; NanoLuc

35 Binary Technology; spike protein; vaccine development, drug development, bioluminescence 
The severe acute respiratory syndrome coronavirus 2 (SARS-CoV-2) is the etiologic agent responsible for the COVID-19 pandemic and is an ongoing worldwide public health threat. As of October $16^{\text {th }} 2020$, there have been over 39 million confirmed cases, and over 1 million confirmed

51 https:/www.who.int/emergencies/diseases/novel-coronavirus-2019/situation-reports).

52 Tremendous efforts are currently underway to develop rapid drug screening methodologies and novel vaccines. The large variability of disease severity among individuals infected with SARS$\mathrm{CoV}-2$ continues to be investigated [1] and new findings in this field may shed light on strategies to tailor these new therapeutics to patients.

Entry of SARS-CoV-2 is mediated by interaction of the viral Spike glycoprotein (S) with its main target receptor angiotensin converting enzyme 2 (ACE2), found on the surface of mammalian cells, primarily in the lower respiratory tract [2]. The $\mathrm{S}$ protein is composed of two subunits, S1 and S2, which play cooperatively a role in viral entry and fusion of the viral membrane with host cell membrane. Binding to ACE2 is mediated by the receptor binding domain (RBD), located in the C-terminus of the S1 subunit [3]. The identification of amino acid residues that are crucial for the interaction of RBD with ACE2 is of great interest to gain a better understanding of the interplay between viral entry and host genetic factors which may contribute to the observed variability in disease pathogenesis. As such, characterizing functional mutants of RBD and ACE2 may provide critical insights for the development of drugs and vaccines.

The development of biosensor technology is a highly valuable and sensitive analytical tool with a broad spectrum of applications, such as diagnosis and drug development $[4,5]$. Biosensors designed to emit bioluminescence often rely on luciferase, a class of enzymes that catalyze substrate to produce a bioluminescent signal. One such tool is the NanoLuc Binary Technology (NanoBiT), which enables rapid analysis of protein-protein interactions through use of

71 Nanoluciferase, a small luciferase reporter [5-12]. By exploiting this technology, we have recently

72 developed an assay to rapidly investigate RBD and ACE2 interactions. In addition, this assay can

73 be used to elucidate the impact of both RBD and ACE2 amino acid mutations on their binding 74 abilities, as well as their potential implications for drug development and evaluating immune 75 responses. 
Previous studies have examined crucial residues in ACE2 and the SARS-CoV-1 Spike domain $[13,14]$. Similarly, recent studies have begun to unravel important interactions in SARSCoV-2 RBD and ACE2 [15]. While some progress has been made towards examining the impact of specific mutations within the SARS-CoV-2 RBD and ACE2, there remain much to be studied with regards to their impact on binding, infectivity and host susceptibility to viral infection. In addition, the impact of specific RBD mutations on the efficacy of potential therapeutics has not been explored. For example, most vaccines under development are designed to contain the RBD domain of $\mathrm{S}$, and sequencing data has shown that $\mathrm{RBD}$ is among the most non-conservative domain in SARS-CoV-2 S [16]. Thus, the question that still remain is whether SARS-CoV-2 viruses harboring select RBD mutations would be controlled by immune responses mounted against the RBD sequence encoded in the original virus strain. Another unexplored area is whether variations in host ACE2 sequences could alter virus susceptibility and/or disease severity among individuals or species. Comparisons on how mutations in ACE2 affect binding of the RBD from SARS-CoV-1 versus SARS-CoV-2 have yet to be performed. We believe that elucidating which variations may have an impact in the ACE2: SARS-CoV-2 S binding affinity is worth investigating since this information could impact the development of therapeutic options for COVID-19 patients. Here, we investigate whether any of these critical amino acid sites in ACE2 exist in the human population and may explain severity of the disease.

\section{Results and Discussion}

The first crucial step of SARS-CoV-2 viral entry is mediated by binding of RBD to ACE2, its main cognate receptor expressed on the surface of the human airway epithelium (Figure 1A). In this study, we aim to investigate whether selected mutations in both SARS-CoV-2 S protein RBD and its host receptor ACE2 could impact their interactions with one another. We also examine how specific mutations in ACE and RBD may alter the efficacy of drugs and neutralizing antibodies being developed for treatment and disease prevention purposes, respectively. To accomplish these aims, we use a NanoBiT SARS-CoV-2-RBD and ACE2 biosensor, previously developed by our lab, to initially characterize the RBD: ACE2 interaction (Figure 1B) [17]. The molecular basis of this technology involves the fusion of a Large Bit ( $\mathrm{LgBiT})$ subunit to one of the proteins of interest, and the fusion of a Small Bit subunit (SmBiT) to the second protein being investigated. As illustrated in Figure 1B, LgBiT and SmBiT alone have poor affinity for one 
106

107

108

109

110

111

112

113

114

115

116

117

118

119

120

121

122

123

124

125

126

127

128

129

130

131

132

133

another; however, both subunits interact to produce a bioluminescent signal in the presence of furimazine, the substrate for Nanoluciferase, if the fusion proteins interact with each other.

\section{Mutations within the ACE2 ectodomain alter binding affinity of ACE2 with SARS-CoV-2} RBD, SARS-CoV-2 S1 subunit, and SARS-CoV-1 RBD

ACE2 is a key interacting partner involved in SARS-CoV-2 viral entry, thus we first performed in silico mutagenesis analysis to assess putative ACE2 mutants that could be potentially defective at interacting with SARS-CoV-2 RBD. Based on the overall 3D crystal structure analysis of ACE2 bound to RBD (Figure 2A, B), we selectively identified 22 sites in ACE2, which are most likely involved in ACE: RBD direct interaction (Figure 2 A-D). To analyze the contribution of these 22 mutants on their ability to interact with RBD using our NanoBiT based biosensor technology, we first engineered the 22 amino acid mutants of ACE2 (Figure 2E) and linked them to SmBiT. Expression of ACE2 mutants from transfected HEK293T cells was demonstrated by immunoblotting (Figure 3A).

We then investigated the binding affinity of ACE2 wild-type and mutants with both SARS$\mathrm{CoV}-2-\mathrm{RBD}$ and SARS-CoV-2 $\mathrm{S} 1$ as complementary binding partner. The rationale for also including an S1-based NanoBiT binding partner in our assays was that RBD is encompassed within the $\mathrm{S} 1$ subunit of the $\mathrm{S}$ glycoprotein. Hence, including $\mathrm{S} 1$ would more closely mimic SARS-CoV-2 $\mathrm{S}$ behavior in the context of viral infection. Thus, we proceeded to compare whether S1 fused to $\mathrm{LgBiT}$ and RBD-LgBiT constructs have similar binding affinity for ACE and its mutants. When combined with SARS-CoV-2-RBD-LgBiT, 12 of the 22 mutants of ACE2 showed a significant decrease in their binding affinity (Figure 3B). Specifically, ACE2 mutants Q24A, F28A, D38A, Y41A, K353A, G354D, D355A, R357A, and NFS (residues 82-84) demonstrated reduced binding to SARS-CoV-2-RBD (Figure 3B). We also found that 13 of the 22 mutants showed a significant decrease in binding to S1 (Figure 3C). Specifically, ACE2 mutants Q24A, F28A, D38A, Y41A, Q42A, L45A, M82A, Y83A, K353A, G354D, D355A, R357A, and NFS had reduced binding to SARS-CoV-2 S1 (Figure 3C). In summary, we found that the same 12 ACE2 mutants that showed decreased binding to SARS CoV-2 RBD also has impaired interactions with SARS CoV-2 S1, with the addition of mutant Y83A that reported a reduced affinity for SARS-CoV-1 S1 only. 
Since structural analysis has shown that certain residues in SARS-CoV-2 RBD are well conserved within SARS-CoV-1 RBD [17], we decided to also combined the various ACE2-SmBiT mutants with a LgBiT-SARS-CoV-1 RBD construct. This allowed us to also evaluate how ACE2 mutations could impact ACE2 : SARS-CoV-1 RBD interaction. In figure 3D, we further show that 14 different ACE2 mutations (T27D, F28A, H34A, E35A, E37A, Y41A, Q42A, L45A, Y83A, K353A, G354D, D355A, R357A, and NFS) significantly alter its respective binding efficiency to the wild type SARS-CoV-1 RBD. Of note, ten of these ACE2 mutants are in common with SARSCoV-2 RBD. These data suggest that in comparison with SARS-CoV-1 RBD, SARS-CoV-2 RBD may be slightly more resistant to ACE2 mutants, which may arise from single nucleotide polymorphisms (SNPs). This observation may have implications with regards to viral susceptibility of various species or individuals based on their ACE2 gene sequence.

SARS-CoV-2 Spike pseudotyped lentiviral particle assay confirm that mutations within the ACE2 ectodomain alter binding affinity of ACE2 with SARS-CoV-2 RBD

To reinforce the ACE2 mutational findings observed with our biosensor technology described above, we then decided to utilize a lentiviral-based pseudovirus infectivity assay. We hypothetized that ACE2 mutants which retain their binding capacity to SARS-CoV-2 RBD should act as competitive inhibitors for SARS-CoV-2 pseudovirus binding to ACE2 in the host cell, and thus reducing its infectivity (Figure 4A). To test this notion, we combined each of the 22 ACE2 mutants with SARS-CoV-2 spike pseudotyped lentivirus expressing luciferase, and then added the pre-treated pseudovirus samples to HEK293X cells expressing ACE2 and TMPRSS2 (the host protease responsible for cleaving $\mathrm{S}$ glycoprotein[18]). We then measured the infectivity with a luciferase assay - a lower signal is indicative of reduced infection, resulting from ACE2 mutants retaining RBD binding capacity, thereby acting as competitive inhibitors (Figure 4B). These results very closely align with mutant binding affinity changes observed with our biosensor technology (Figure 3B-C).

\section{Utilizing ACE2 sequences as a predictor for the SARS-CoV-2 susceptibility of various} species and mutation prevalence in humans

Our findings suggest that identification of ACE2 amino acid sites which impact RBD binding may provide insights as to SARS-CoV-2 susceptibilities of different individuals or species 
163

164

165

166

167

168

169

170

171

172

173

174

175

176

177

178

179

180

181

182

183

184

185

186

187

188

189

190

191

based on their genetics. Using multiple sequence alignments of the 12 target mutation sites identified to be essential for RBD binding (Figure 3), we propose a method to predict SARS-CoV2 virus susceptibility (Figure 5A). It is plausible to hypothetize that any species with an identical ACE2 amino acid sequence to the human ACE2 receptor would theoretically be highly susceptible to the virus, and possibly act as a source of transmission, such as the common chimpanzee (Pan troglodytes; Figure 5A). With the presence of only one or two differences at key ACE2 binding sites, it is likely that species such as Tursiops truncatus (bottlenose dolphin) or Cricetulus griseus (Chinese hamster) would still be infected by SARS-CoV-2. In contrast, species such as domesticated horses (Equus caballus) or brown rats (Rattus norvegicus), which have many differences at key ACE2 residues, are expected to be less susceptible to SARS-CoV-2 infection, due to reduced binding efficiency between ACE2 and RBD. With reduced binding efficacy, viral infection and spread would likely be impaired in these animals, leading them to pose less of a risk for shedding large amounts of infectious virus potentially leading to reduced viral pathogenesis (Figure 5A). These findings are consistent with literature studies of optimal SARS-CoV-2 disease models [19]. For example, increasing studies have indicated that hamsters are a better suited animal model to study SARS-CoV-2 in comparison to mice [20].

Looking at the prevalence of these mutations in people, we observe that some of these SNPs (namely NFS, G354, and D355) already exist in a small fraction of the population (Figure 5B). Our data suggest the possibility that individuals harboring these SNPs may be more resistant to the SARS-CoV-2 virus infection. Genetic differences in the ACE2 receptor of individuals may also provide a partial explanation for the variability in disease severity among individuals. Interestingly, in silico studies from other groups have also suggested that genetic influences cause interindividual variability in ACE2 expression [21], [22]. In this study, we experimentally validated for the first time the effects of ACE2 mutants on RBD binding affinity, which may pave the path for clinical studies.

Mutations in SARS-CoV-2 RBD that alter its ability to bind to ACE2 do not impact the activity of a competitive inhibitor nor the efficacy of a neutralizing antibody 
A key concern surrounding RNA viruses, and viruses in general, is their capacity to evolve through the accumulation of mutations. Such mutations may drive resistance to therapeutics and/or evasion of host immune responses acquired through infection or vaccination against the wild-type virus. Recently two mutations in the SARS-CoV-2 RBD have been reported in Africa and the UK. We have investigated one of the critical mutation sites reported by the UK, asparagine residue at location 501 (N501), by altering the residue to both to Alanine and Tyrosine in silico (Figure 6 A-D). The change to alanine preserved the internal interactions of the residue with two glutamine residues at positions 498 and 506 but abolished the interaction with tyrosine 41 in ACE2. However, mutation to the tyrosine variant preserved all internal interactions and slightly strengthened them. Moreover, this mutation introduced a new strong interaction site, 2.3 A, with lysine 353 in ACE2 structure. Another recently discovered mutation is the change of glutamic acid at position 484 to lysine. We did not detect any significant change in internal or RBD-ACE2 interaction sites which may be due to the fact that this residue is located in a very flexible loop structure (Figure 6E-F).

some level of ACE2 binding capacity to further assess the effects of a competitive inhibitor and a neutralizing antibody with our biosensor assay. From a therapeutic perspective, recombinant RBD is under clinical consideration for use as a potential viral attachment inhibitor, given its ability to competitively inhibit SARS-CoV-2 virus particles [24]. We found that recombinant RBD expressed from mammalian cells wascapable of out-competing all 16 of the RBD mutants tested(Figure 6I). These results suggest that competitive drugs developed to combat the wild-type virus are still applicable to virus strains which may acquire these mutations in the RBD domain leading to reduced receptor binding over time. Neutralizing antibodies $(\mathrm{nAb})$ play a key role in generating protective immunity against viral agents, and are developed through exposure to the virus itself, or to viral antigens in the form of a vaccine. We found that addition of a $\mathrm{nAb}$ significantly decreased, if not abolished, the interaction between all examined RBD mutants and ACE2 (Figure 6J). Importantly, this data may indicate that these receptor binding mutations do not provide an immune evasive advantage to the virus, and as a result, should not pose a serious threat to the efficacy of developed vaccines, and immunity, to viruses harboring these mutations. 


\section{Conservation of RBD across coronavirus strains}

222

223

224

225

226

227

228

229

230

231

232

233

234

235

236

237

238

239

240

241

242

243

244

245

246

247

248

249

250

We used multiple sequence alignment tools to further investigate amino acid conservation patterns of the RBD mutants under examination across multiple betacoronavirus strains. This analysis could provide interesting insights on the possible contributions of these specific residues in RBD and their link to infectivity. The variation in amino acid sites is indicative of which amino acids are critical for maintaining the structural integrity of RBD, and which can be modified for therapeutic purposes. For example, amino acid C361 is present in the bottom of the RBD core, and not at the contact site. Considering the cross-species conservation of this residue, it may exert an important role in stabilizing the overall structure of the protein. Of the analyzed amino acids, K417 was the only one which was not part of RBM, yet is still in close proximity to the contact site, providing important contact with residue D30 in the ACE2 structure [3]. Of the other Betacoronavirus species analyzed in this study, the presence of K417 is limited to SARS-CoV-2. This unique event could be aone of the possible reason for which SARS-CoV-2 exhibits a higher binding efficiency to ACE2 compared to other viruses in the same family. It is also likely to expect that Betacoronaviruses harboring low amino acid sequence similarity to SARS-CoV-2 likely utilize a different host receptor for viral entry. In addition, when analyzing tyrosine residues at positionY449 and Y505, it seems that the hydroxyl $(\mathrm{OH})$ group in the tyrosine side chain may possibly contact two different amino acids on ACE2 structure, appearing critical for effective binding of the receptor-ligand structure. Precisely, Y449 contacts D38 and Q42 in ACE2, and Y505 contacts E37 and R393 in ACE2, respectively [3].

Elucidating which amino acids are conserved and occupy critical roles in viral ligand-host receptor binding could deepen our understanding of antiviral drug development. To increase the spectrum of antiviral agents directed towards betacoronaviruses, targeting conserved amino acids which are crucial for receptor binding may prove to be an interesting strategy. Given the conservation of amino acids between SARS-CoV-2, SARS-CoV-1 and MERS, it would be of interest to develop a therapeutics targeting this triad of viruses.

It has been theorized that the SARS-CoV-2 virus originated from bats, was then transmitted to pangolins, and finally spilled over to humans [25], [26], [27]. We examined the conservation of the $25 \mathrm{RBD}$ point mutants generated in our study to determine if variations in amino acid sequence would follow the predicted path of interspecies transmission. Interestingly, these sites were mostly 
251

252

253

254

255

256

257

258

259

260

261

262

263

264

265

266

267

268

269

270

271

272

273

274

275

276

277

conserved across all three species, with only three sites (V445, F486, and Y505) differing in bat $\mathrm{CoV}$, when compared to pangolin Co-V and human SARS-CoV-2, and only one site (K417) differing in pangolin CoV compared to the bat and human viruses (Figure 7). These observations are in line with a recent report by Liu et al. [27]. In this study, the authors aligned the full-length spike sequence of SARS-CoV-2, Pangolin-CoV and Bat-CoV-RaTG13. These results and our predictions reported herein support the hypothesis that the path of viral transmission originated from bats, continued to pangolins as intermediate hosts, and then followed its dispersal route to the human population.

\section{Conclusions}

In this study, we provide for the first time a comprehensive overview of the effects of select mutations in both the ectodomain of ACE2 and the RBD of SARS-CoV-2. Our findings suggest that ACE2 SNPs in the human population may account for the variability in infectivity and disease progression in the context of SARS-CoV-2 infection. Furthermore, our observations from RBD mutational scanning also provide potential sites for drug targeting and therapeutic development. By combining ACE2 and RBD mutational analyses, we provide insights into the genetics underlying virus susceptibility and the development of possible therapeutic targets.

\section{Materials and Methods}

\subsection{Cell lines}

HEK293T (ATCC ${ }^{\circledR}$ CRL-3216) human embryonic kidney cells were obtained from ATCC. Cells were maintained in Dulbecco's Modified Eagle's Medium (Gibco), supplemented with 10\% FBS (VWR) and 1\% penicillin/streptomycin (Invitrogen). Cells were incubated in a humidified $37^{\circ} \mathrm{C}$ incubator at $5 \% \mathrm{CO}_{2}$. Cells are routinely tested for mycoplasma by PCR testing, and used for up to 20 passages after thawing.

\subsection{Plasmids}

Engineered inserts are outlined in Supplementary Table 1 (GenScript). All biosensor subunits were cloned into the BamHI/NotI sites of pcDNA3.1 to generate mammalian expression constructs.

\subsection{Transient transfection}


HEK293T cells were grown in $100 \mathrm{~mm}$ or $150 \mathrm{~mm}$ cell culture dishes to $70 \%$ confluence. Cells were transfected with $10 \mu \mathrm{g}$ of DNA using PolyJet (SignaGen) according to manufacturer's protocol. 48 hours post-transfection, cell supernatants or cell lysates were harvested for subsequent testing.

\subsection{In vitro NanoBit assay}

293T cells were lysed using passive lysis buffer (Promega). NanoBiT assays were performed using native coelenterazine (CTZ; 3.33 mM final concentration; Nanolight Technologies - Prolume Ltd., Pinetop, AZ, USA). Synergy Microplate Reader (BioTek, Winooski, VT, USA) was used to measure luminescence. Results are presented as raw RLU (Relative Luminescence Unit), or normalized to control where indicated. The data presented are the mean of three biological replicates.

\subsection{Western immunoblotting}

293T cells were lysed using passive lysis buffer. Protein concentration in clarified whole cell lysates was quantified using bicinchoninic acid assay (BCA) (Pierce). $20 \mu \mathrm{g}$ of total protein was prepared in 1x Laemmli buffer, loaded into 4-12\% gradient Bis-Tris acrylamide gels and resolved. Following transfer onto nitrocellulose membrane, blots were incubated in $0.1 \%$ Ponceau S solution (in $5 \% \mathrm{v} / \mathrm{v}$ acetic acid-distilled water) for 5 minutes at room temperature, imaged, then washed with TBST for 10 minutes. Blots were then blocked using 5\% skimed milk powder in TBST for 1 hour. Blots were subsequently probed using anti-beta-actin primary antibody (1:5000), anti-HA tag $(1: 1000)$, or anti-FLAG tag $(1: 1000)$ in $5 \%$ milk overnight at $4^{\circ} \mathrm{C}$. Following washing with TBST, membranes were probed with HRP-conjugated secondary antibodies (1:10000) in 5\% milk at room temperature for 1 hour. Blots were washed with TBST, then developed using BioRad Clarity-Western ECL and the BioRad ChemiDoc imaging system.

\subsection{Lentiviral Pseudovirus Assay}

Plasmid encoding SARS-CoV-2 spike pseudotyped lentivirus was kindly provided by Dr. Jesse Bloom (Fred Hutchinson Cancer Research Center, Seattle, WA) and lentivirus was generated as previously described (Crawford et. al., 2020). HEK293T cells were seeded in 6 wells plates to $70 \%$ confluency and were transfected with lug of individual smBiT ACE2 mutant DNA using 
PolyJet (SignaGen) according to manufacturer's protocol. 48 hours post-transfection, supernatants of transfected cells were harvested. Pseudotyped lentiviruses were incubated with each of the smBiT-ACE2 mutant containing supernatants for 1 hour at $37^{\circ} \mathrm{C}$. Immediately following the incubation period, polyclonal HEK293X cells stably overexpressing ACE2 and TMPRSS2 were transduced in 96 well format (300 000 cells). 4 hours post-transduction all media was replaced. 48 hours post-transduction, cells were lysed using 1 X PLB, cell lysates were transferred to an opaque 96-well plate, and infection efficiency was measured by luciferase assay using the Bright-Glo Luciferase Assay system following manufacturer's protocols (Promega).

\subsection{Statistical analysis}

All graphs and statistical analyses were generated in Excel or GraphPad Prism v.8. Means of two groups were compared using two-tailed unpaired Student's t-test. Means of more than two groups were compared by one-way ANOVA with Dunnett's or Tukey's multiple comparisons correction. Alpha levels for all tests were 0.05 , with a $95 \%$ confidence interval. Error is graphed as the standard deviation (SD). Measurements were taken from distinct samples (biological replicates). For all analyses, ${ }^{*} \mathrm{P}<0.05, * * \mathrm{P}<0.01, * * * \mathrm{P}<0.005$, n.s. $=$ not significant.

\subsection{Multiple Sequence Alignment of ACE2 and Spike Sequences, and finding ACE2 SNPs in}

\section{the target positions}

All of the represented sequences were retrieved from NCBI refseq protein database. Multiple sequence alignment (MSA) was performed using Clustal Omega (Madeira et.al., 2019) algorithm with default parameters. Alignments were visualized and prepared for illustration using Jalview software (Waterhouse et.al., 2009). The multiple sequence alignment of ACE2 polypeptides was reordered based on the binding efficiency data in the J. Damas et al (Damas et.al., 2020). The NCBI dbSNP database was searched for possible single nucleotide polymorphisms (SNPs) in target mutation sites and the related information were gathered in a table using Microsoft Excel.

\section{Funding:}

The authors disclosed receipt of the following financial support for the research, authorship, and/or publication of this article: This work was funded by the generous support from the Ottawa Hospital Foundation and a grant from the Canadian Institutes of Health Research (\#448323) to 
334 J.C.B., C.S.I. and J-S.D. This work was also supported by a Fast Grant for COVID-19 Science to

335 J.C.B. and C.S.I. as well as an Ontario Together Grant to J-S-D. T.A. is funded by a CIHR

336 Banting Fellowship. R.S. is funded by a CIHR postdoctoral fellowship and a cluster MITACs

337 fellowship. T.R.J. is funded by a CIHR Frederick Banting and Charles Best Canada and

338 Graduate Scholarship and an Ontario Graduate Scholarship. E.E.F.B. is funded by a CIHR

339 Frederick Banting and Charles Best Canada Graduate Scholarship and a Lebovic Fellowship.

340 A.S. and S.T. were supported by NSERC Graduate Scholarships. M.J.F.C., J.D., S.B., N.T.M.,

341 J.P., J.T.W, J.D. and S.T. are funded by cluster MITACs fellowships. Finally, we would like to

342 thank the excellent technical assistance of Xiaohong He, Ricardo Marius, Julia Petryk and

343 Christiano Tanese De Souza.

344 Conflicts of Interest: The authors declare no conflict of interest

Supplemental Table 1

\begin{tabular}{|c|c|c|}
\hline Insert & DNA sequence & $\begin{array}{c}\text { Amino Acid } \\
\text { sequence }\end{array}$ \\
\hline \multirow{2}{*}{ SmBiT-ACE2 } & ATGTGCCCCCAAAAGTTGACCATCTC & MCPQKLTIS \\
& ATGGTTCGCAATTGTACTACTAGTGA & WFAIVLLVS \\
& GTCCCTTGATGGCAGGCGGAAGTGGA & PLMAGGSG \\
\hline
\end{tabular}




\begin{tabular}{|c|c|c|}
\hline \multirow{31}{*}{$\begin{array}{c}\text { (IL12 secretion signal-ACE2- } \\
\text { HA-His) }\end{array}$} & GGTTCCTCTGGAGTGACCGGATACAG & GSSGVTGYR \\
\hline & GCTGTTCGAGGAGATCCTGCAGAGCA & LFEEILQSTIE \\
\hline & CAATCGAGGAGCAGGCCAAGACCTT & EQAKTFLDK \\
\hline & CCTGGACAAGTTTAACCACGAGGCCG & FNHEAEDLF \\
\hline & AGGATCTGTTTTATCAGAGCTCCCTG & YQSSLASWN \\
\hline & GCCTCCTGGAACTACAATACCAACAT & YNTNITEEN \\
\hline & CACAGAGGAGAATGTGCAGAACATG & VQNMNNAG \\
\hline & AACAATGCCGGCGACAAGTGGTCTGC & DKWSAFLKE \\
\hline & СТTCCTGAAGGAGCAGAGCACACTGG & QSTLAQMYP \\
\hline & CCCAGATGTACCCTCTGCAGGAGATC & LQEIQNLTV \\
\hline & CAGAACCTGACCGTGAAGCTGCAGCT & KLQLQALQQ \\
\hline & GCAGGCCCTGCAGCAGAATGGCTCTA & NGSSVLSED \\
\hline & GCGTGCTGTCCGAGGATAAGTCTAAG & KSKRLNTIL \\
\hline & CGGCTGAATACAATCCTGAACACCAT & NTMSTIYST \\
\hline & GAGCACAATCTATTCCACCGGCAAGG & GKVCNPDNP \\
\hline & TGTGCAATCCCGACAACCCTCAGGAG & QECLLLEPG \\
\hline & TGTCTGCTGCTGGAGCCTGGCCTGAA & LNEIMANSL \\
\hline & TGAGATCATGGCCAACAGCCTGGATT & DYNERLWA \\
\hline & ACAATGAGAGGCTGTGGGCATGGGA & WESWRSEV \\
\hline & GTCCTGGCGCTCTGAAGTGGGCAAGC & GKQLRPLYE \\
\hline & AGCTGCGGCCACTGTACGAGGAGTAT & EYVVLKNE \\
\hline & GTGGTGCTGAAGAACGAGATGGCCA & MARANHYE \\
\hline & GAGCCAATCACTACGAGGACTATGGC & DYGDYWRG \\
\hline & GATTACTGGAGGGGCGACTATGAGGT & DYEVNGVD \\
\hline & GAACGGCGTGGACGGCTACGATTATA & GYDYSRGQL \\
\hline & GCCGCGGCCAGCTGATCGAGGATGTG & IEDVEHTFEE \\
\hline & GAGCACACATTCGAGGAGATCAAGC & IKPLYEHLH \\
\hline & CCCTGTATGAGCACCTGCACGCCTAC & AYVRAKLM \\
\hline & GTGCGGGCCAAGCTGATGAATGCCTA & NAYPSYISPI \\
\hline & TCCAAGCTACATCTCCCCAATCGGAT & GCLPAHLLG \\
\hline & GCCTGCCTGCACACCTGCTGGGCGAC & DMWGRFWT \\
\hline
\end{tabular}




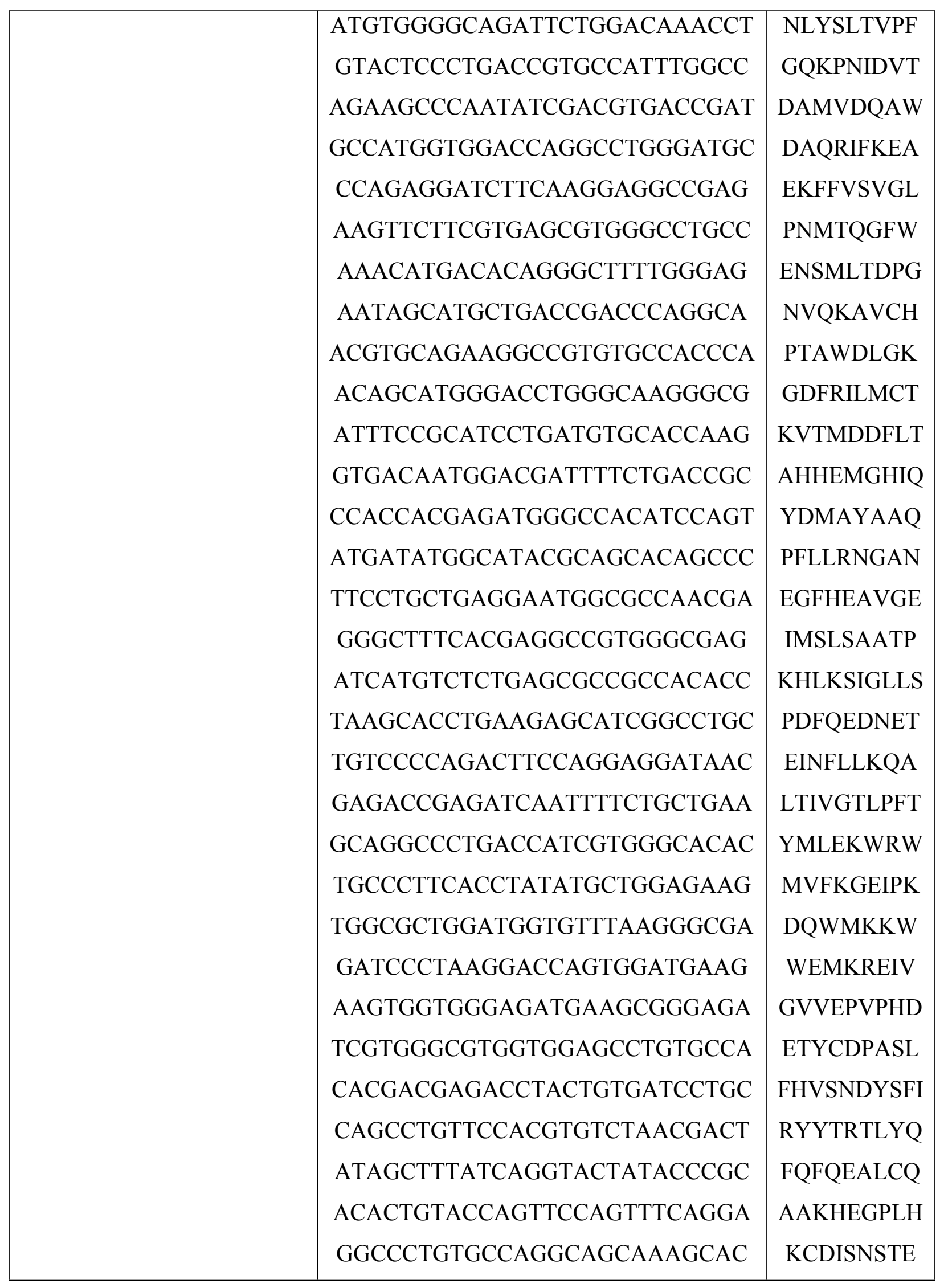




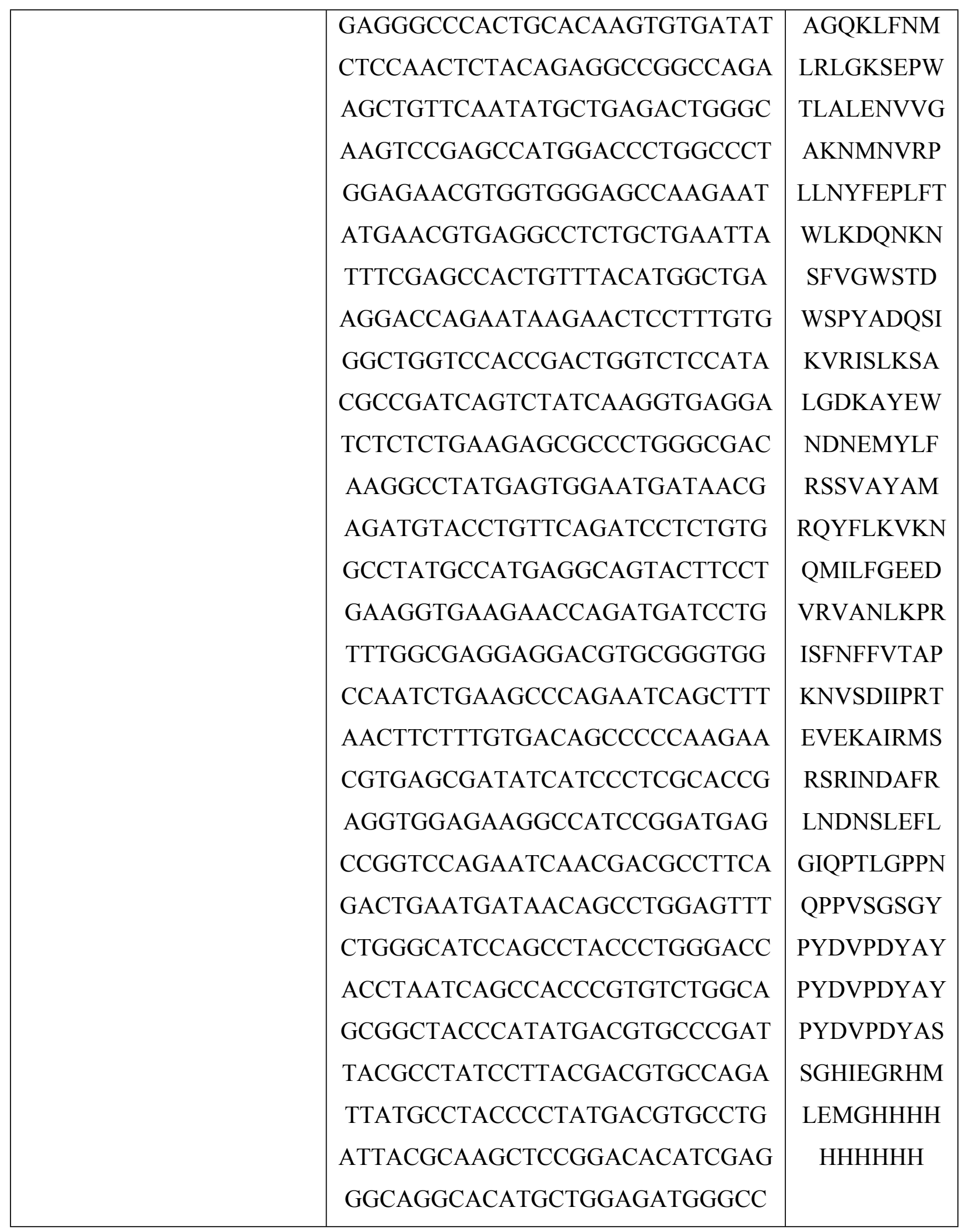




\begin{tabular}{|c|c|c|}
\hline & $\begin{array}{c}\text { ATCATCACCATCATCACCACCACCAC } \\
\text { CACTGA }\end{array}$ & \\
\hline $\begin{array}{c}\text { RBD-LgBiT } \\
\text { (IgK leader-3XFLAG-RBD- } \\
\text { LgBiT-His) }\end{array}$ & $\begin{array}{l}\text { ATGGAGACAGACACACTCCTGCTATG } \\
\text { GGTACTGCTGCTCTGGGTTCCAGGTT } \\
\text { CCACTGGTGACTCTGGCTCTAGCGGC } \\
\text { TCTGGCAGCGGCGACTACAAGGACC } \\
\text { ACGACGGTGACTACAAGGACCACGA } \\
\text { CATCGACTACAAGGACGACGACGAC } \\
\text { AAGGGAGGAGGAGGCTCTAGCGGCG } \\
\text { GCAACATCACAAATCTGTGCCCATTC } \\
\text { GGCGAGGTGTTTAACGCCACCAGATT } \\
\text { TGCCAGCGTGTATGCCTGGAACCGGA } \\
\text { AGAGAATCTCTAATTGCGTGGCCGAC } \\
\text { TATAGCGTGCTGTACAATAGCGCCTC } \\
\text { CTTCTCTACCTTTAAGTGCTATGGCGT } \\
\text { GTCCCCCACAAAGCTGAACGACCTGT } \\
\text { GCTTCACCAACGTGTACGCCGACTCT } \\
\text { TTTGTGATCAGGGGCGATGAGGTGCG } \\
\text { CCAGATCGCACCTGGACAGACAGGC } \\
\text { AAGATCGCCGACTACAACTATAAGCT } \\
\text { GCCAGACGATTTCACCGGCTGCGTGA } \\
\text { TCGCCTGGAATAGCAACAATCTGGAT } \\
\text { TCCAAAGTGGGCGGCAACTACAATTA } \\
\text { TCTGTACCGGCTGTTCAGAAAGAGCA } \\
\text { ACCTGAAGCCCTTTGAGCGGGATATC } \\
\text { AGCACAGAGATCTACCAGGCAGGCT } \\
\text { CCACCCCTTGCAACGGAGTGGAGGGC } \\
\text { TTCATTGTATTTTCCCCTGCAGAGC }\end{array}$ & $\begin{array}{c}\text { METDTLLL } \\
\text { WVLLLWVP } \\
\text { GSTGDSGSS } \\
\text { GSGSGDYKD } \\
\text { HDGDYKDH } \\
\text { DIDYKDDDD } \\
\text { KGGGGSSGG } \\
\text { NITNLCPFGE } \\
\text { VFNATRFAS } \\
\text { VYAWNRKR } \\
\text { ISNCVADYS } \\
\text { VLYNSASFS } \\
\text { TFKCYGVSP } \\
\text { TKLNDLCFT } \\
\text { NVYADSFVI } \\
\text { RGDEVRQIA } \\
\text { PGQTGKIAD } \\
\text { YNYKLPDDF } \\
\text { TGCVIAWNS } \\
\text { NNLDSKVG } \\
\text { GNYNYLYR } \\
\text { LFRKSNLKP } \\
\text { FERDISTEIY } \\
\text { QAGSTPCNG } \\
\text { VEGFNCYFP } \\
\text { LQSYGFQPT } \\
\text { RGVYQPY } \\
\text { RYSFL }\end{array}$ \\
\hline
\end{tabular}




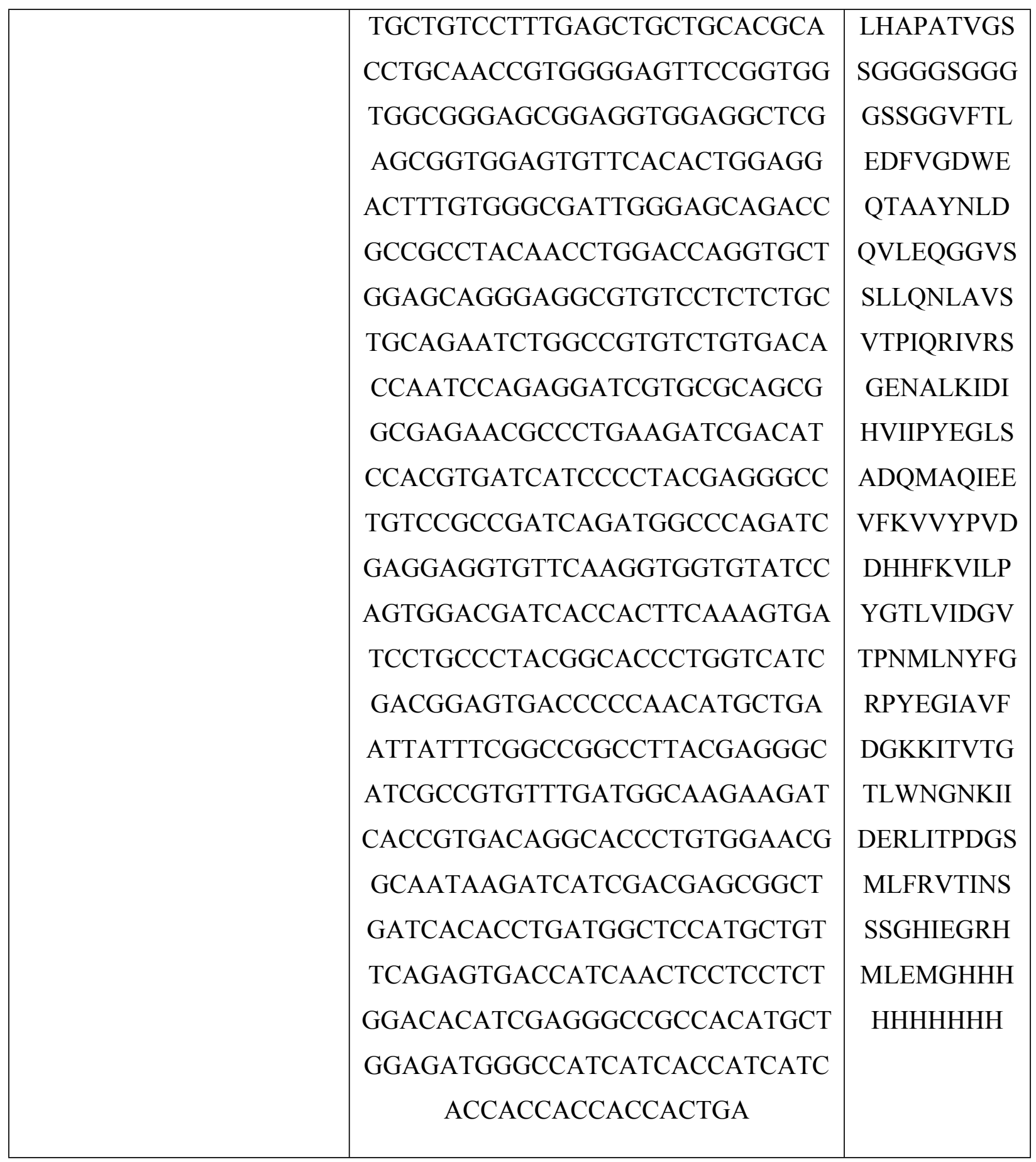


1. Casanova, J.L., H.C. Su, and C.H.G. Effort, A Global Effort to Define the Human Genetics of Protective Immunity to SARS-CoV-2 Infection. Cell, 2020. 181(6): p. 1194-1199.

2. Hikmet, F., et al., The protein expression profile of ACE2 in human tissues. Mol Syst Biol, 2020. 16(7): p. e9610.

3. Lan, J., et al., Structure of the SARS-CoV-2 spike receptor-binding domain bound to the ACE2 receptor. Nature, 2020. 581(7807): p. 215-220.

4. Vigneshvar, S., et al., Recent Advances in Biosensor Technology for Potential Applications - An Overview. Front Bioeng Biotechnol, 2016. 4: p. 11.

5. Azad, T., A. Tashakor, and S. Hosseinkhani, Split-luciferase complementary assay: applications, recent developments, and future perspectives. Anal Bioanal Chem, 2014. 406(23): p. 5541-60.

6. Azad, T., et al., A LATS biosensor screen identifies VEGFR as a regulator of the Hippo pathway in angiogenesis. Nat Commun, 2018. 9(1): p. 1061.

7. Azad, T., et al., A gain-of-functional screen identifies the Hippo pathway as a central mediator of receptor tyrosine kinases during tumorigenesis. Oncogene, 2020. 39(2): p. 334-355.

8. Nouri, K., et al., Identification of Celastrol as a Novel YAP-TEAD Inhibitor for Cancer Therapy by High Throughput Screening with Ultrasensitive YAP/TAZ-TEAD Biosensors. Cancers (Basel), 2019. 11(10).

9. Nouri, K., et al., A kinome-wide screen using a NanoLuc LATS luminescent biosensor identifies ALK as a novel regulator of the Hippo pathway in tumorigenesis and immune evasion. FASEB J, 2019. 33(11): p. 12487-12499.

10. Yang, X., T. Azad, and K. Nouri, Hippo Pathway Bioluminescent Biosensor. 2019, US Patent App. $16 / 177,691$.

11. England, C.G., E.B. Ehlerding, and W. Cai, NanoLuc: a small luciferase is brightening up the field of bioluminescence. Bioconjugate chemistry, 2016. 27(5): p. 1175-1187.

12. Dixon, A.S., et al., NanoLuc complementation reporter optimized for accurate measurement of protein interactions in cells. ACS chemical biology, 2016. 11(2): p. 400-408.

13. Han, D.P., A. Penn-Nicholson, and M.W. Cho, Identification of critical determinants on ACE2 for SARS-CoV entry and development of a potent entry inhibitor. Virology, 2006. 350(1): p. 15-25.

14. Li, W., et al., Receptor and viral determinants of SARS-coronavirus adaptation to human ACE2. EMBO J, 2005. 24(8): p. 1634-43.

15. Li, Q., et al., The Impact of Mutations in SARS-CoV-2 Spike on Viral Infectivity and Antigenicity. Cell, 2020. 182(5): p. 1284-1294 e9.

16. Chen, J., et al., Mutations strengthened SARS-CoV-2 infectivity. arXiv preprint arXiv:2005.14669, 2020.

17. Azad, T., et al., Nanoluciferase complementation-based biosensor reveals the importance of $\mathrm{N}$ linked glycosylation of SARS-CoV-2 Spike for viral entry. 2020.

18. Hoffmann, M., et al., SARS-CoV-2 cell entry depends on ACE2 and TMPRSS2 and is blocked by a clinically proven protease inhibitor. Cell, 2020.

19. Mohandas, S., et al., Evaluation of the susceptibility of mice \& hamsters to SARS-CoV-2 infection. The Indian Journal of Medical Research, 2020. 151(5): p. 479.

396 20. Mohandas, S., et al., Evaluation of the susceptibility of mice \& hamsters to SARS-CoV-2 infection. 
21. Brest, P., et al., Host Polymorphisms May Impact SARS-CoV-2 Infectivity. Trends Genet, 2020. 36(11): p. 813-815.

400

22. Cao, Y., et al., Comparative genetic analysis of the novel coronavirus (2019-nCoV/SARS-CoV-2)

401 receptor ACE2 in different populations. Cell Discov, 2020. 6: p. 11.

402

403

23. Starr, T.N., et al., Deep Mutational Scanning of SARS-CoV-2 Receptor Binding Domain Reveals Constraints on Folding and ACE2 Binding. Cell, 2020. 182(5): p. 1295-1310 e20.

404

24. Tai, W., et al., Characterization of the receptor-binding domain (RBD) of 2019 novel coronavirus:

405 implication for development of RBD protein as a viral attachment inhibitor and vaccine. Cell Mol Immunol, 2020. 17(6): p. 613-620.

407

25. Lam, T.T., et al., Identifying SARS-CoV-2-related coronaviruses in Malayan pangolins. Nature, 2020. 583(7815): p. 282-285.

409

410

26. Zhang, T., Q. Wu, and Z. Zhang, Probable Pangolin Origin of SARS-CoV-2 Associated with the COVID-19 Outbreak. Curr Biol, 2020. 30(7): p. 1346-1351 e2.

411 27. Liu, P., et al., Are pangolins the intermediate host of the 2019 novel coronavirus (SARS-CoV-2)? 
Figure Legends:

416 Figure 1. Schematic of SARS-CoV-2 viral entry and the NanoBiT biosensor. (A) Schematic

417 of SARS-CoV-2 viral entry via the interaction between the receptor binding domain (RBD) of

418 the spike glycoprotein and the host cell receptor ACE2. (B) Illustration of the NanoBiT

419 complementation-based biosensor which detects interactions between RBD fused to $\operatorname{LgBiT}$ and

420 ACE2 fused to SmBiT.

421

Figure 2. ACE2 amino acids in 3D structure of the bound RBD and ACE2 and schematic representation of mutations in ACE2. (A) 3D illustration of the overall structure of RBD bound to ACE2. RBD is colored in green, receptor-binding motif (RBM) of RBD is in dark blue, and ACE2 is in dark cyan. (B) Enlarged view of the overall structure depicting ACE2 target mutation sites in stick rendition. Sticks are represented in yellow color. (C-D) ACE2 target mutation sites in stick representation at the contact site of two molecules. Dotted lines connect the mutant AAs to their contacting AAs in RBM. The structure is from PDB: 6M0J based on the information provided in Lan et al. 2020 (E) Illustration of the amino acid changes used to examine ACE2 in this study. and viral determinants of ACE2-RBD interaction. (A) Immunoblot of SmBiT-ACE2 mutant expression from the cell lysates of transfected HEK293T cells. $\beta$-actin and total protein loading are shown as controls. (B-D) Biosensor assay with SmBiT-ACE2 mutants and (B) LgBiT-SARS CoV-2 RBD, (C) LgBiT-SARS CoV-2 Spike S1, or (D) LgBiT-SARS-CoV-1 RBD demonstrating altered binding affinity of various mutants. critical host and viral determinants of ACE2-RBD interaction. (A) Schematic of the lentiviral SARS-CoV-2 pseudovirus infectivity assay demonstrating spike-pseudotyped lentiviral assay demonstrating the capacity of each mutant examined to act as a competitive inhibitor for the spike-host ACE2 interaction. 

Mutants. (A) Sequences were reordered based on their binding efficiency to RBD. Only the target mutation sites are shown and the non-target amino acids (AA) has been cut out from the alignment representation. The alignment coloring scheme is based on the chemical properties of each AA (each Human AA is green, the AA from the same chemistry is orange, and AA with different chemistry is red. Except for the last column with three AAs which the number of identical AAs to the human determines the color. WebLogo (http://weblogo.threeplusone.com) representation shows a relative scale of the presence of each AA in each column. (B) dbSNP data on single nucleotide Polymorphism in the ACE2 nucleotide sequence on the target amino acid codons. The frequency of each SNP in different datasets was gathered in the last column. A synonymous mutation (no effect on the protein sequence) is green and missense mutations are red.

Figure 6. (A-F) Visualization of RBD N501 and E484 mutations. (A) overall structure of RBD and ACE2 interaction. (B) the loop structure in RBD containing E484 residue. (C) Predicted structure following E484 residue mutation to lysine which would not significantly change the nature of its interaction in the loop structure of RBD, (D) the N501 residue and its interacting residues in RBM and ACE2. (E) Predictedstructure when the N501 residue mutates to Alanine which would eliminate ACE2 interaction but preserve internal RBM interactions. (F) Predicted structure when the N501 residue mutates to tyrosine which would preserve and slightly boosts internal interactions and introduces an stronger interaction site with ACE2 compared to the wild type residue. (G) ACE2 demonstrating altered binding affinity of various mutants of RBD-LgBiTmutants.(H) Immunoblot of RBD-LgBiT mutant expression from the cell lysates of transfected HEK293T cells. $\beta$-actin and total protein loading are shown as controls. Competition biosensor assay with LgBiT-RBD mutants which maintained any binding capacity pre-incubated with (I) recombinant $\mathrm{RBD}$ protein or $(\mathrm{J})$ neutralizing antibody to examine therapeutic implications of various mutants. mutation sites are shown and the non-target amino acids (AA) has been cut out from the alignment 471 representation. The alignment coloring scheme is based on the chemical properties of each AA 472 (each SARS-CoV-2 AA is green, the AA from the same chemistry is orange, and AA with different 473 chemistry is red. WebLogo (http://weblogo.threeplusone.com) representation shows a relative 
474 scale of the presence of each AA in each column. (B) Cross species comparison of RBD amino

475 acids in SARS-CoV-2, Pangolin-CoV 2020 and Bat-CoV-RaTG13. Selected mutants are identified

476 by colored boxes. Colored changes indicate RBD AA differences (V445A and F486A and

477 Y505A).

478

479

480

481

482

483

484

485

486 
A

A
Neutralizing antibody

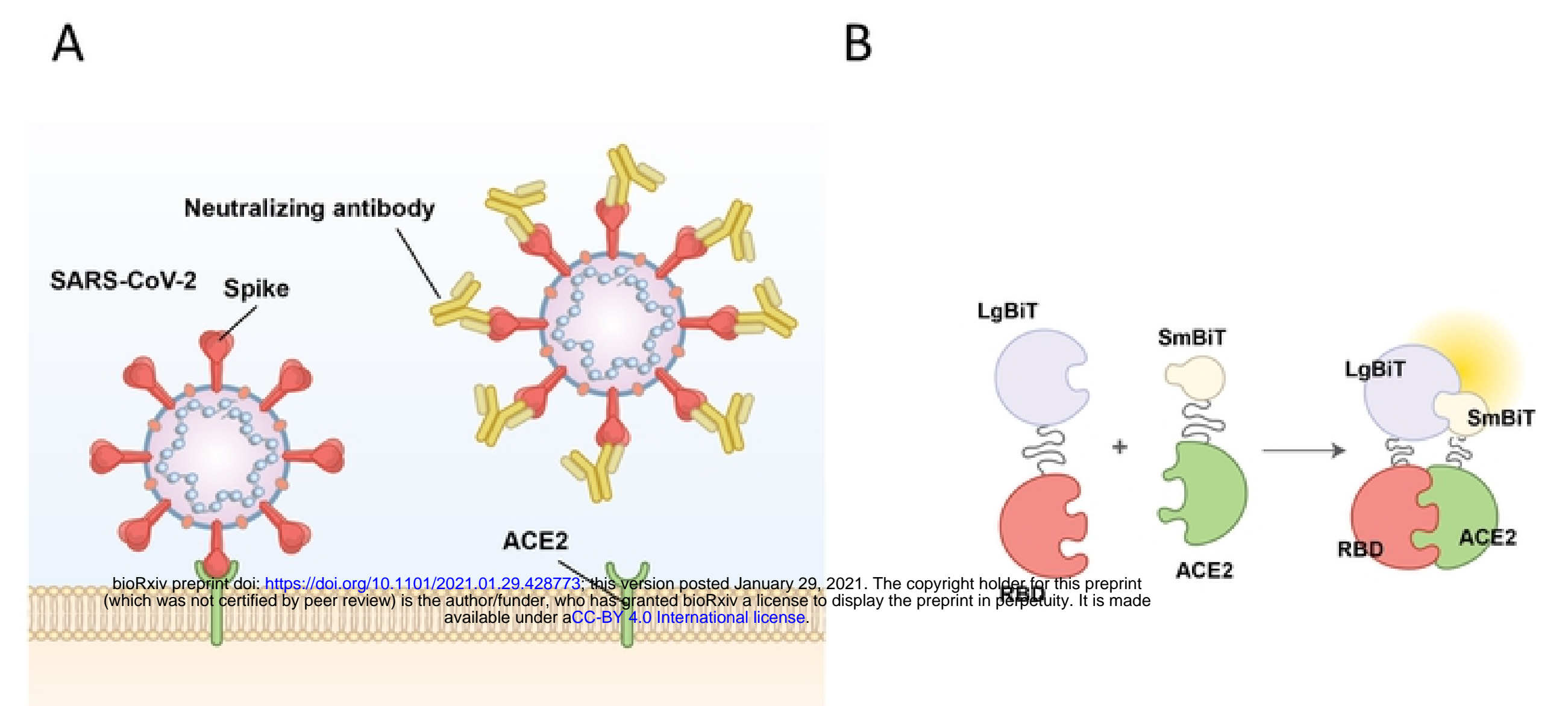

Neutralizing antibody
SARS-CoV-2 Spike

\section{Figure 1}

A

.

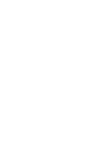

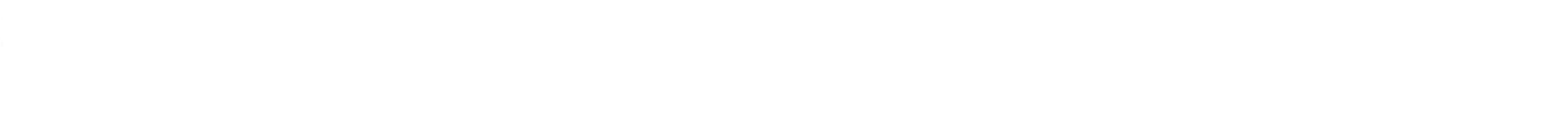

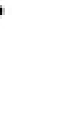

A
Neutralizing antibody
ARS-CoV-2 Spike
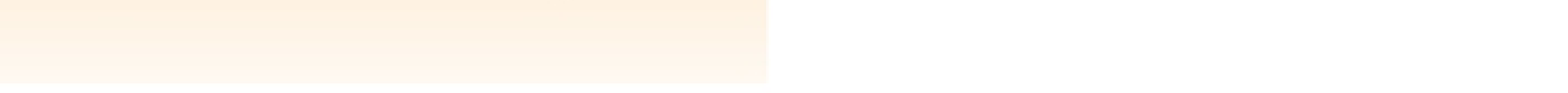

.
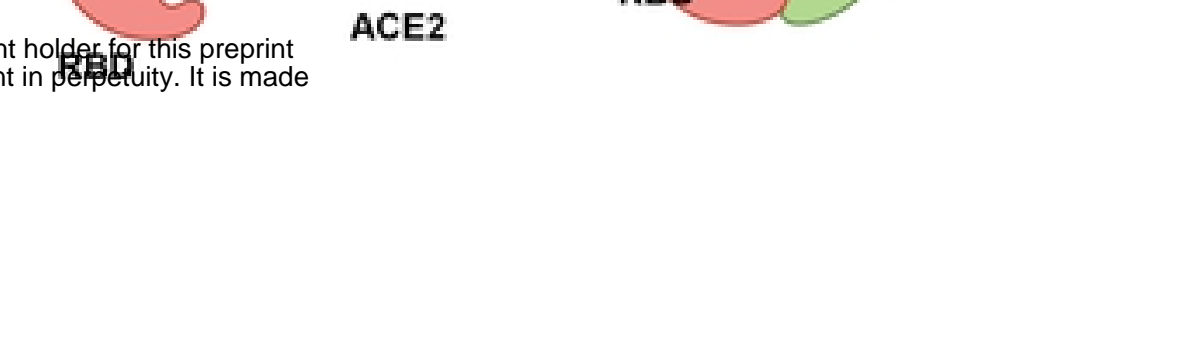


\section{Figure 2}

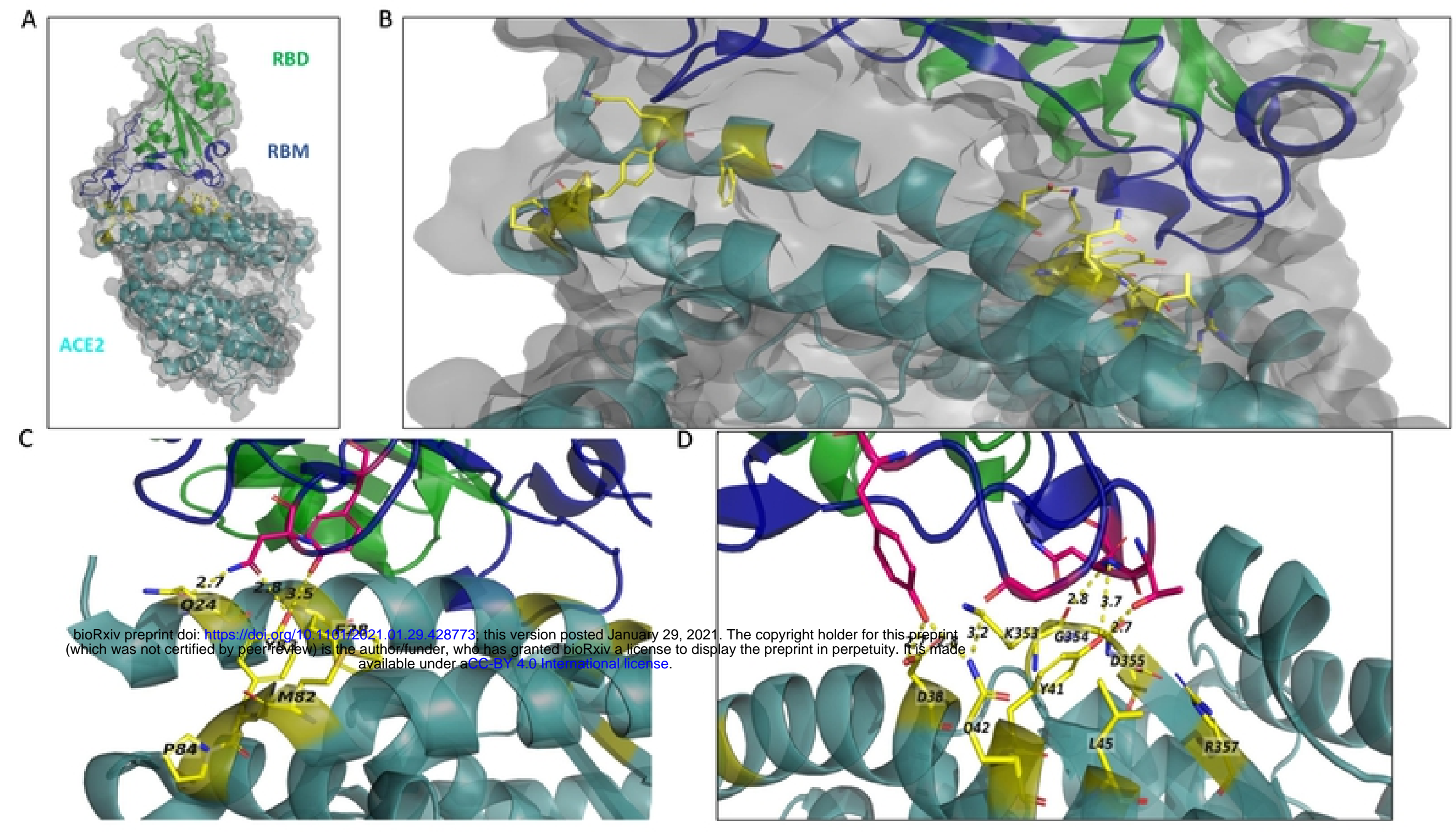

E

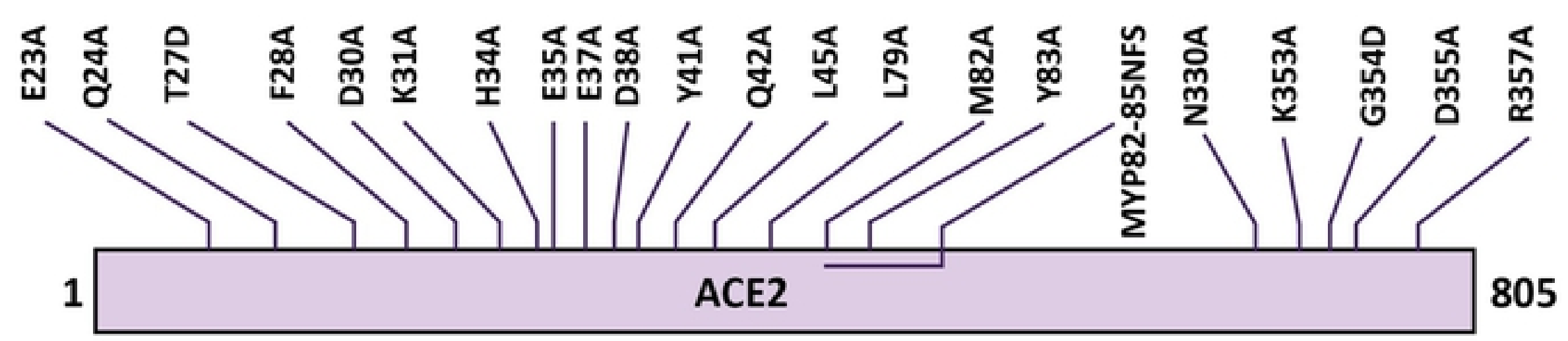


Figure 3

A

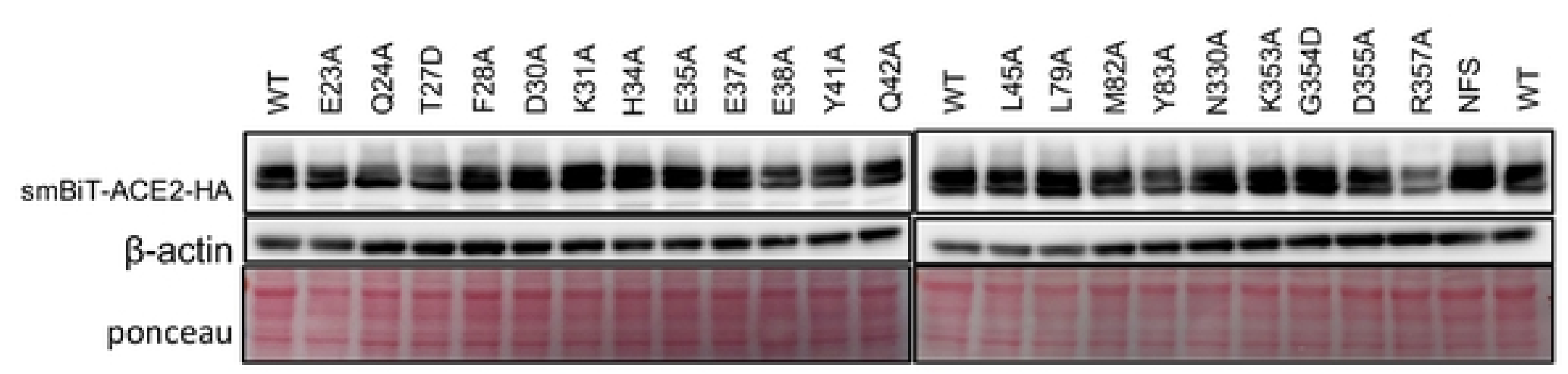

B

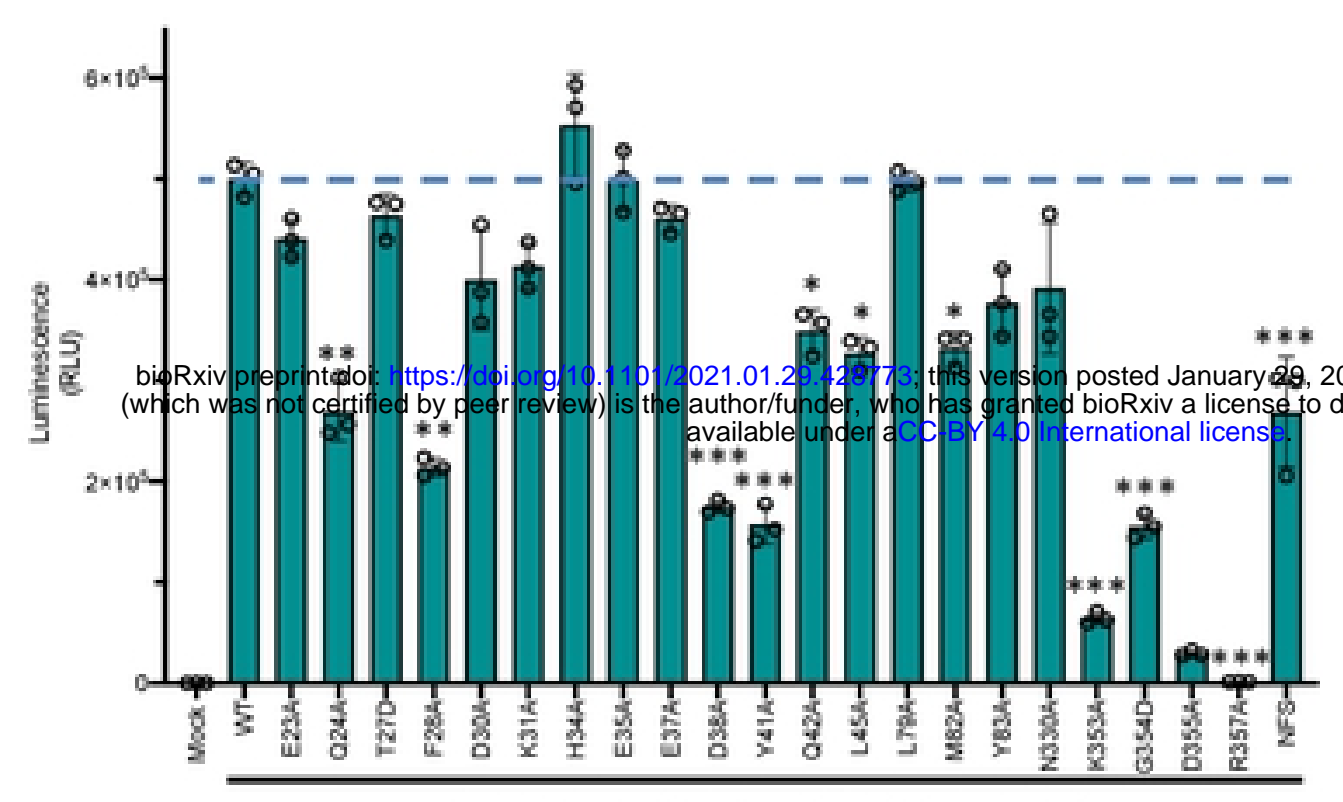

SARS-CoV-2-RBD NanoBiT (ACE2 mutants)
C

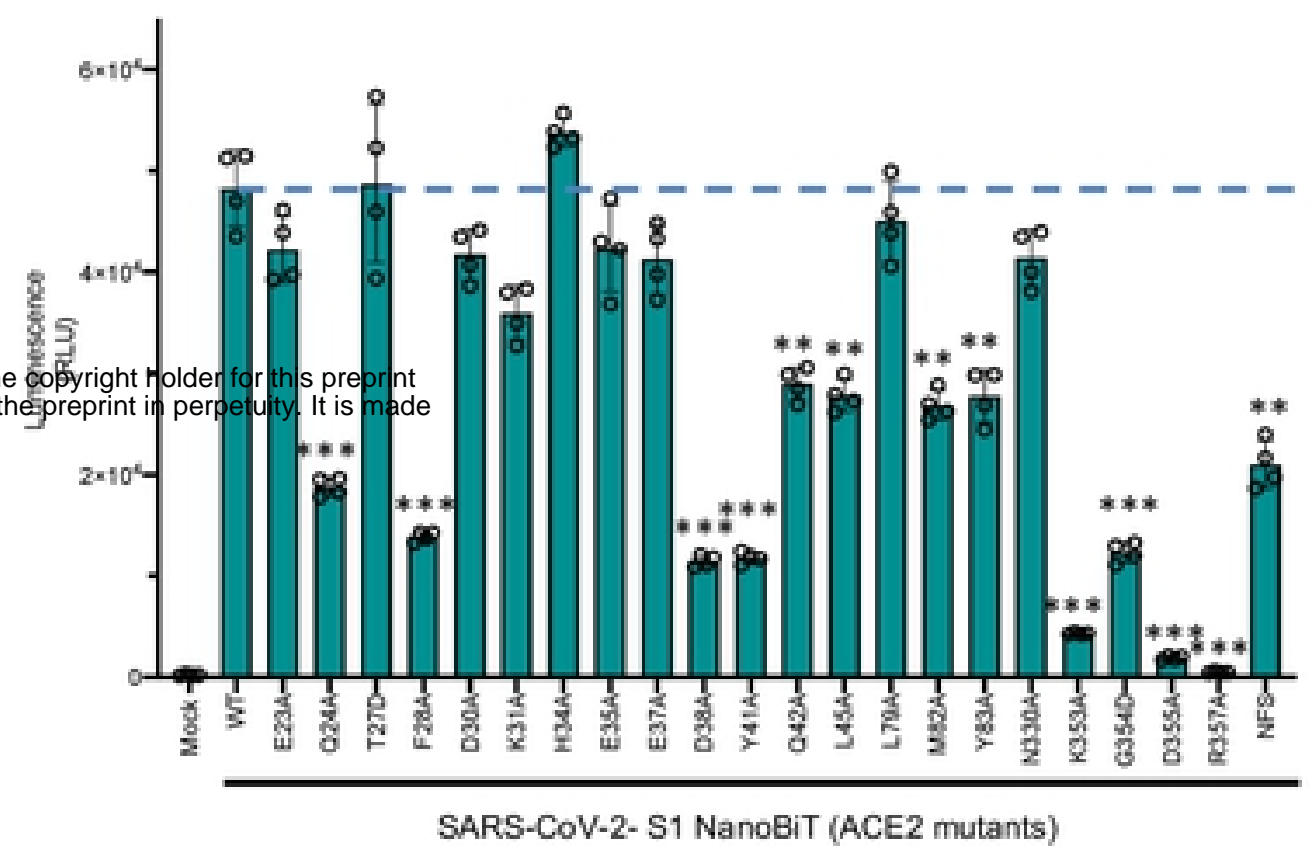

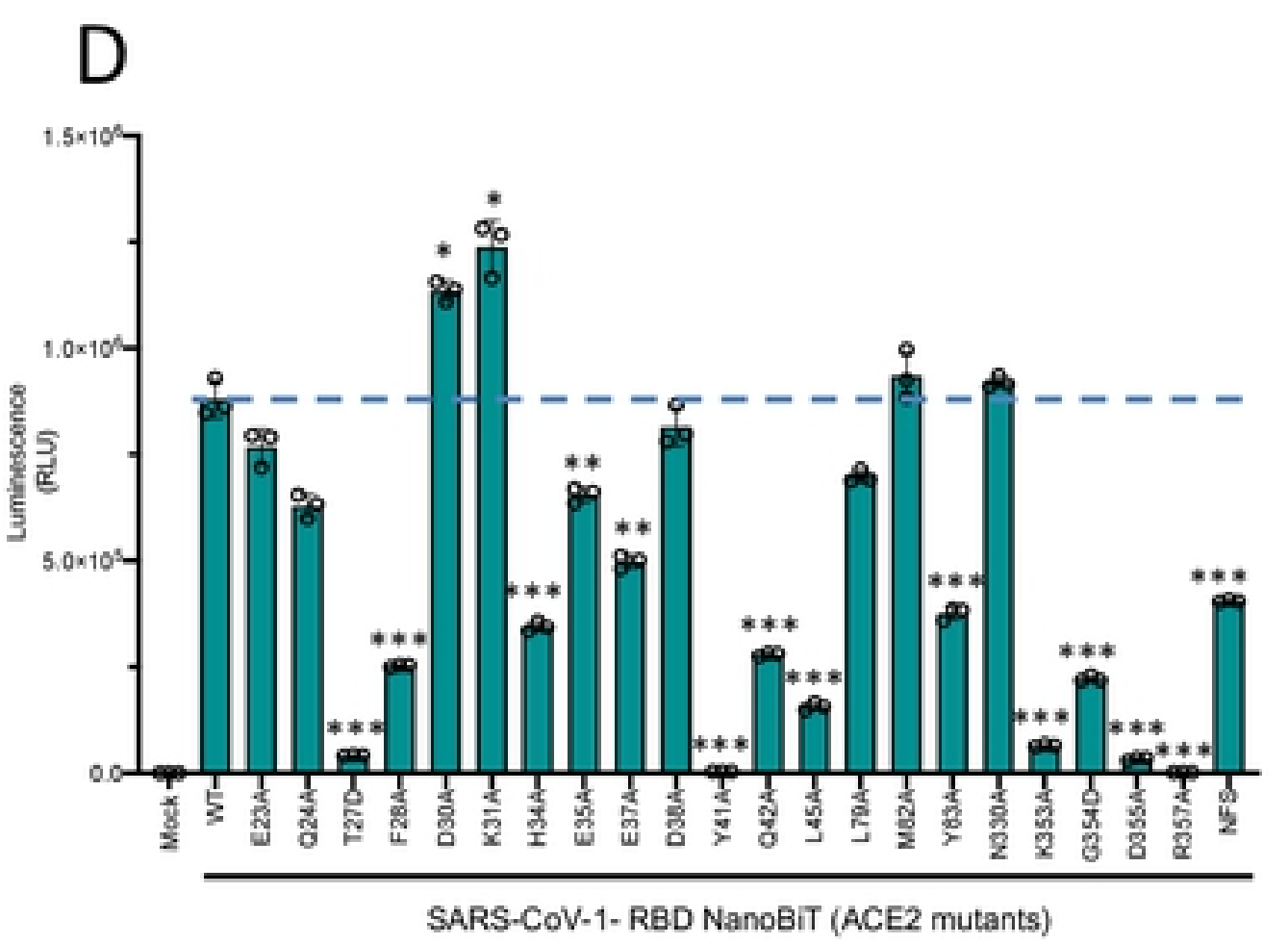


A

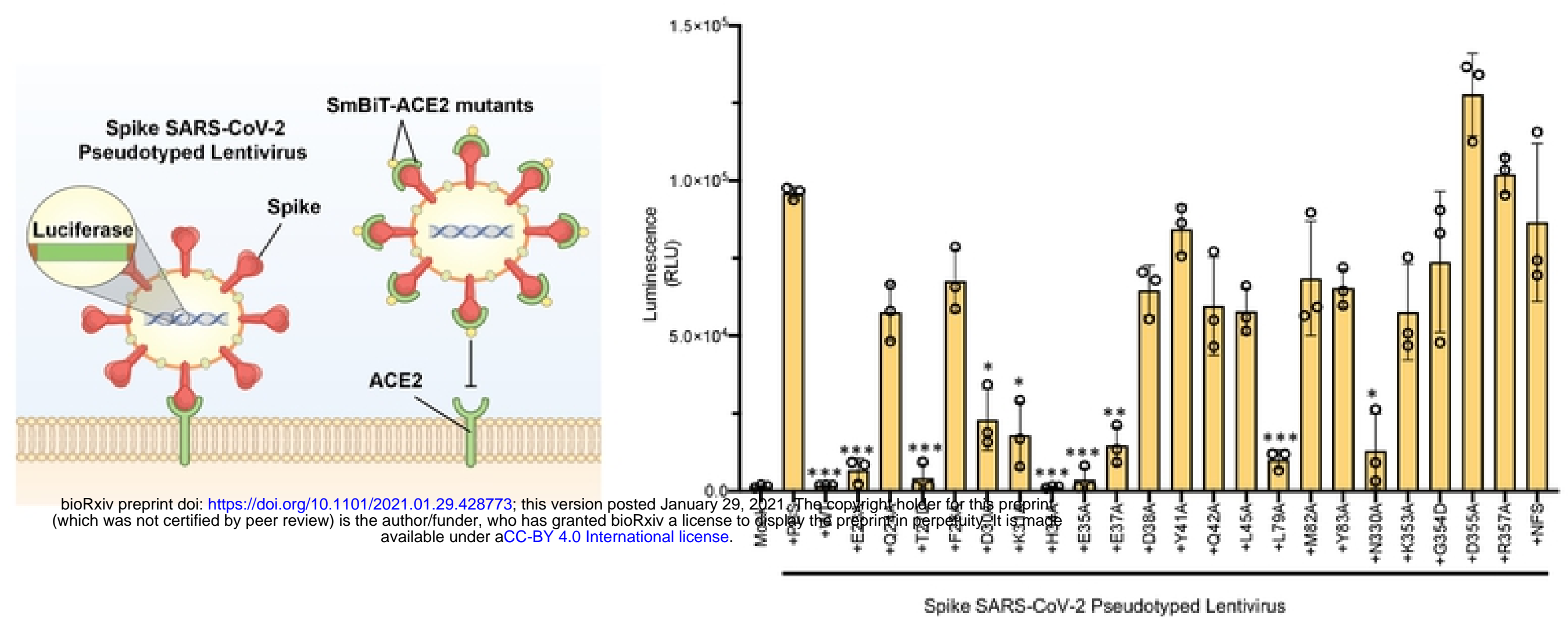

B

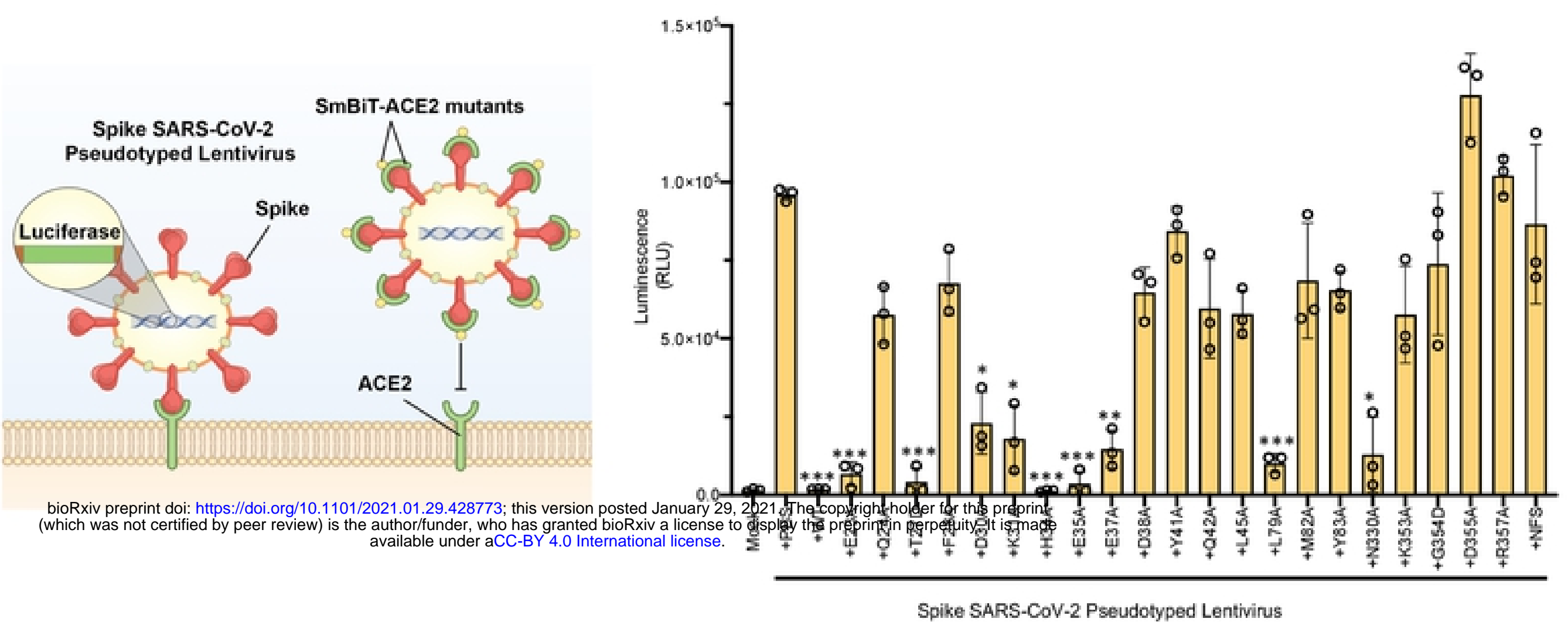

Spike SARS-CoV-2 Pseudotyped Lentivirus 


\section{Figure 5}

A

\begin{tabular}{|c|c|c|c|c|c|c|c|c|c|c|c|c|c|c|c|}
\hline \multicolumn{3}{|c|}{ Multiple Sequence Alignment } & \multicolumn{13}{|c|}{ Mutations } \\
\hline Binomial Name & Common Name & Binding Efficiency & Q24A & F28A & D38A & Y41A & $\mathrm{Q} 42 \mathrm{~A}$ & L45,A & M82A & Y83A & K353A & G354D & D355A & $\mathrm{R} 357 \mathrm{~A}$ & MYP82-85NFS \\
\hline Howo sopviens & Human & 5 & Q & $\mathrm{F}$ & D & Y & Q & L & M & $\mathrm{Y}$ & K & G & D & $\mathbf{R}$ & MYP \\
\hline Pan uroglodines & Chimpanzee & 5 & Q & $\mathrm{F}$ & D & Y & Q & L & M & $Y$ & K & G & D & R & MYP \\
\hline Tirsiogs monsonis & Dolghin & 4 & $\mathbf{R}$ & $\mathrm{F}$ & D & $\mathrm{Y}$ & Q & $\mathrm{L}$ & $\mathrm{T}$ & $\mathrm{Y}$ & $\mathrm{K}$ & G & D & $\mathbf{R}$ & TYP \\
\hline Cricenthes grisens & Hamster & 4 & Q & $\mathrm{F}$ & D & Y & Q & L & N & $Y$ & K & G & D & R & NYS \\
\hline Ovis aries & Sheep & 3 & Q & $\mathrm{F}$ & D & Y & Q & L & $\mathrm{T}$ & $Y$ & K & G & D & R & TYS \\
\hline Felis cants & Cat & 3 & L & $\mathrm{F}$ & $\mathrm{E}$ & $\mathrm{Y}$ & Q & L & $\mathrm{T}$ & $\mathrm{Y}$ & K & G & D & $\mathrm{R}$ & TYP \\
\hline Canis hopus fawillaris & Dog & 2 & L & $\mathrm{F}$ & E & Y & Q & L & $\mathrm{T}$ & $Y$ & K & G & D & R & TYP \\
\hline Eques caballhs & Horse & 2 & L & $\mathrm{F}$ & E & H & Q & L & $\mathrm{T}$ & Y & K & G & D & R & TYP \\
\hline Ses sange & $\mathrm{Pig}_{\mathrm{i}}$ & 2 & $\mathbf{L}$ & $\mathrm{F}$ & D & Y & Q & L & $\mathrm{T}$ & $Y$ & K & G & D & R & TYP \\
\hline Mass moroculas & Mouse & 1 & N & $\mathrm{F}$ & D & Y & Q & L & $\mathrm{S}$ & F & H & G & D & $\mathrm{R}$ & SFS \\
\hline Rames nonszicas & Fin & 1 & $\mathrm{~K}$ & $\mathrm{~F}$ & D & $Y$ & Q & $\mathrm{L}$ & $\mathrm{N}$ & $\mathrm{F}$ & $\mathrm{H}$ & G & D & $\mathrm{R}$ & NFS \\
\hline
\end{tabular}

B

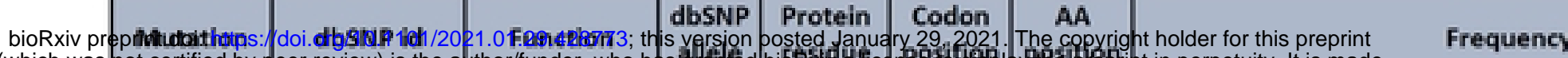
(which was not certified by peer review) is the author/funder, who has tranted bibRXit a ficense to displaythe breprint in perpetuity. It is made

\begin{tabular}{|c|c|c|c|c|c|c|c|}
\hline $\begin{array}{l}\text { enlâtudeititiotps: } \\
\text { not certified by }\end{array}$ & $\begin{array}{l}\text { doi.dibsitol } 1 \text { idh } / 20 \\
\text { peer review) is the a }\end{array}$ & 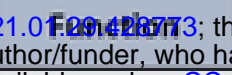 & $\begin{array}{l}\text { s yorsion } \\
\text { s yrianted }\end{array}$ & 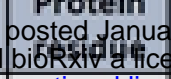 & $\begin{array}{l}\text { ry } 29.2021 . \\
\text { nse totodispia }\end{array}$ & $\begin{array}{l}\text { The copyrio } \\
\text { Ththe propt }\end{array}$ & $\begin{array}{l}\text { ght holder for this preprint } \\
\text { riht in perpetuity. It is made }\end{array}$ \\
\hline Q24A & - & ailable under aCC- & BY 4.0 Int & prnational lice & nse. & - & - \\
\hline F28A & - & - & - & - & - & - & - \\
\hline D38A & $=$ & - & $=$ & $=$ & $=$ & - & - \\
\hline Y41A & - & - & - & - & - & - & - \\
\hline Q42A & - & - & - & - & - & - & - \\
\hline L45A & - & - & - & - & - & - & - \\
\hline \multirow{5}{*}{ NFS (MYP) } & \multirow{3}{*}{ rs766996587 } & reference & G & Met [M] & 3 & 82 & \multirow{3}{*}{$\begin{array}{c}\mathrm{T}=0.000011(2 / 182758, \text { GnomAD_exome }) \\
T=0.000048 \text { (6/125568, TOPMED) } \\
T=0.00001(1 / 87698, \text { ExAC }) \\
T=0.00014 \text { (3/22011, GnomAD) } \\
T=0.0000(0 / 2188, \text { ALFA Project })\end{array}$} \\
\hline & & missense & $\mathrm{T}$ & Ile [I] & 3 & 82 & \\
\hline & & missense & A & Ile [I] & 3 & 82 & \\
\hline & \multirow{2}{*}{ rs759134032 } & reference & C & Pro $[\mathrm{P}]$ & 1 & 84 & \multirow{2}{*}{$\begin{array}{c}T=0.000005(1 / 182792, \text { GnomAD_exome }) \\
T=0.00001(1 / 87705, \text { ExAC })\end{array}$} \\
\hline & & missense & A & Thr [T] & 1 & 84 & \\
\hline K353A & - & - & $=$ & - & $=$ & $=$ & - \\
\hline \multirow{2}{*}{ G354D } & \multirow{2}{*}{ rs200909433 } & reference & C & Gly [G] & 3 & 354 & \multirow{2}{*}{$A=0.000016(2 / 125568$, TOPMED $)$} \\
\hline & & synonymous & $\mathrm{T}$ & Gly [G] & 3 & 354 & \\
\hline \multirow{2}{*}{ D355A } & \multirow{2}{*}{ rs961360700 } & reference & G & Asp [D] & 1 & 355 & \multirow{2}{*}{$\begin{array}{c}T=0.000012 \text { (2/170310, GnomAD_exome) } \\
T=0.0001 \text { (1/8986, ALFA Project) }\end{array}$} \\
\hline & & missense & A & Asn $[\mathrm{N}]$ & 1 & 355 & \\
\hline R357A & - & - & - & - & - & - & - \\
\hline
\end{tabular}



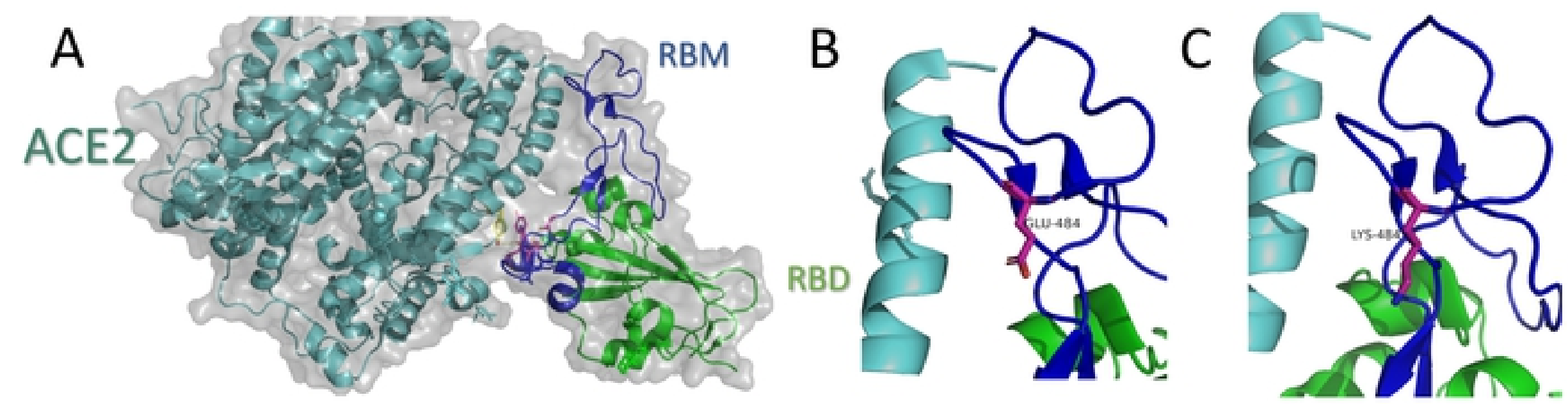

D
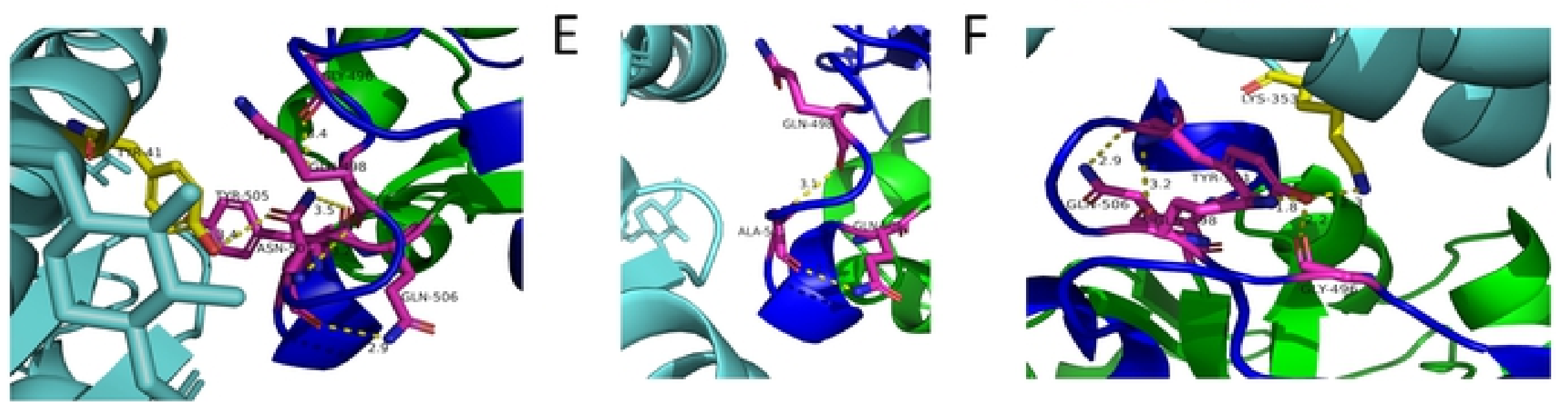

bioRxiv preprint doi: https://doi.org/10.1101/2021.01.29.428773; this version posted Janupry 29,2021 . The copyright holder for this preprint
(whichth was not certified by peer review) is the author/funder, who has granted bioRxiv a liofy to display the preprint in perpetuity. It is made
available under aCC-BY 4.0 International license.
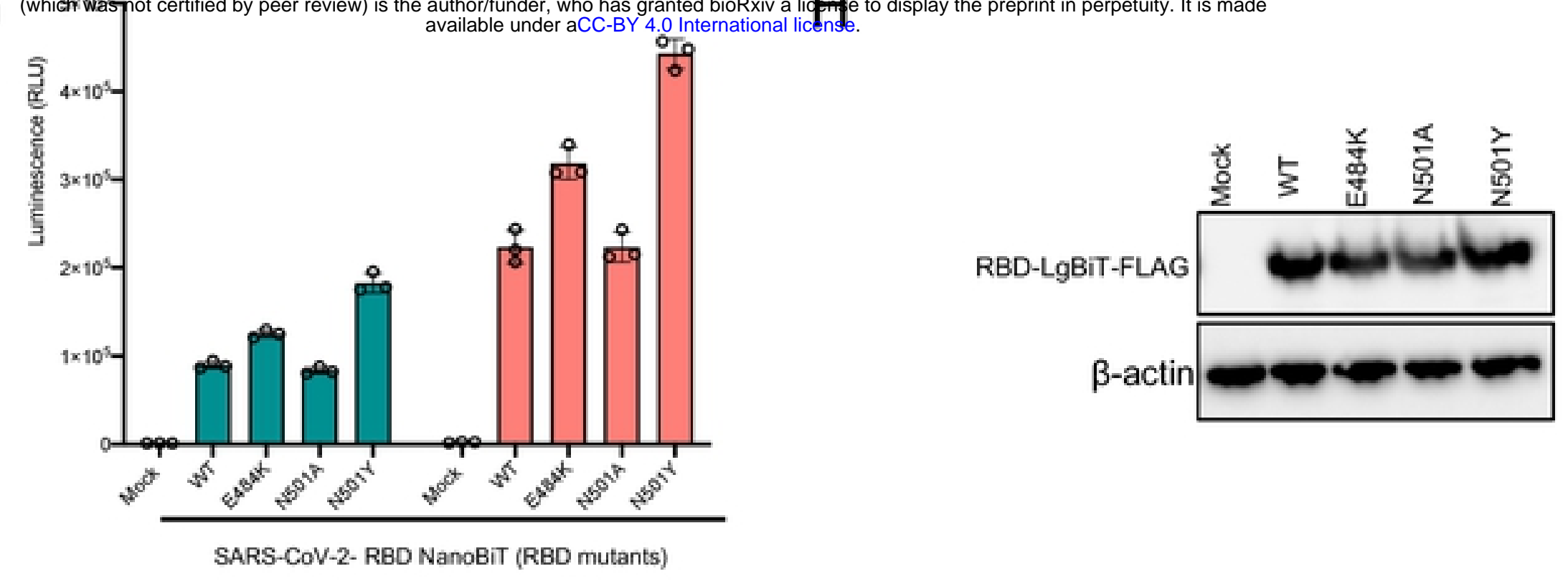

RBD SARS-Cov-2 mutants- ACE2 (RBD competition)

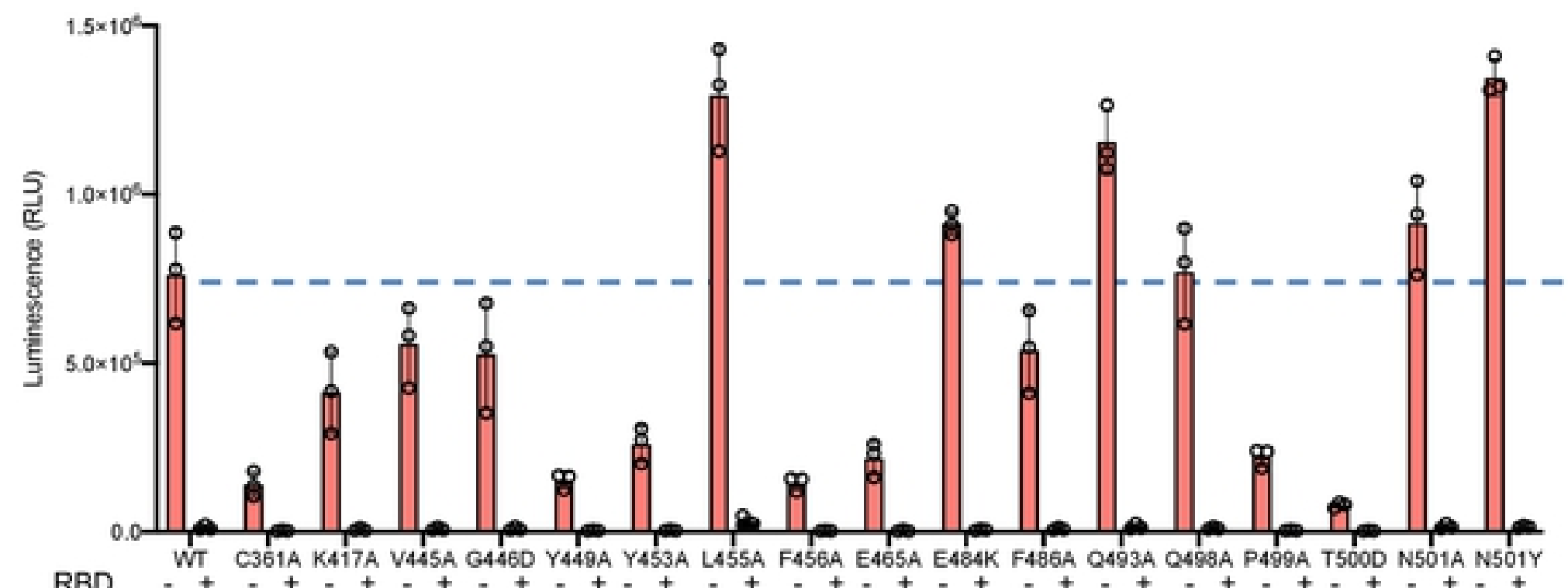

J

SARS-CoV-2-RBD NanoBiT (RBD mutants)

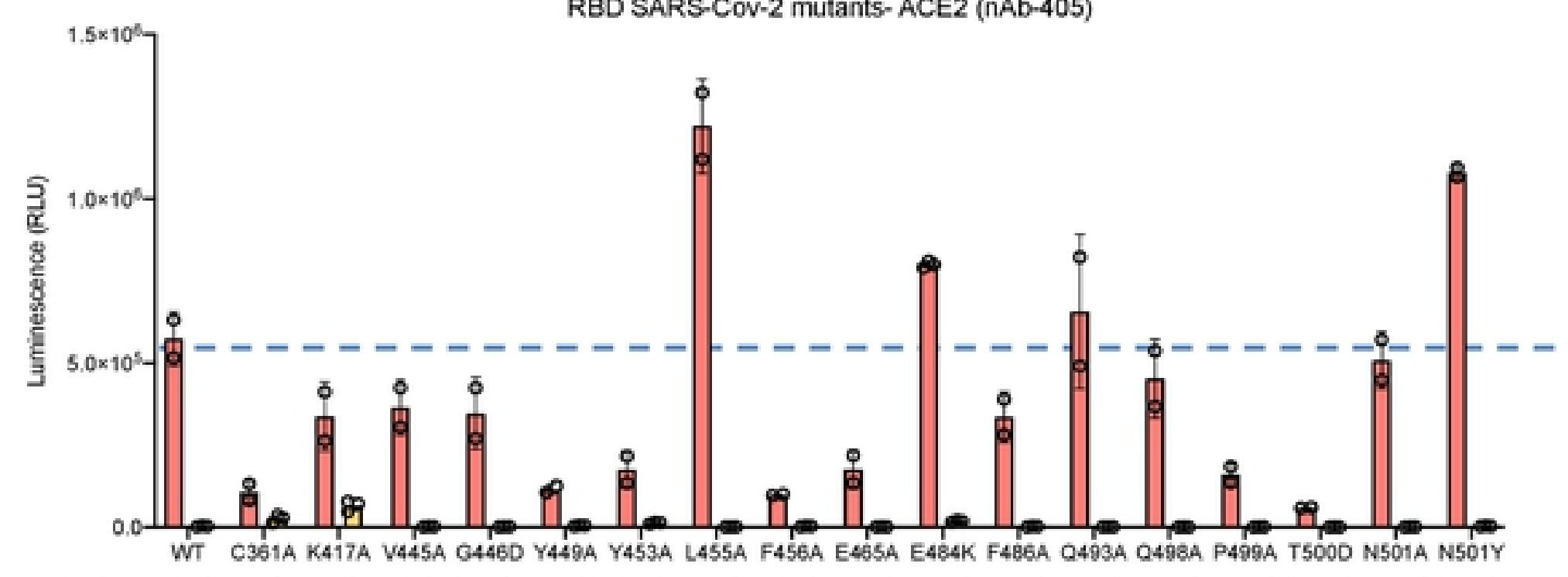


A

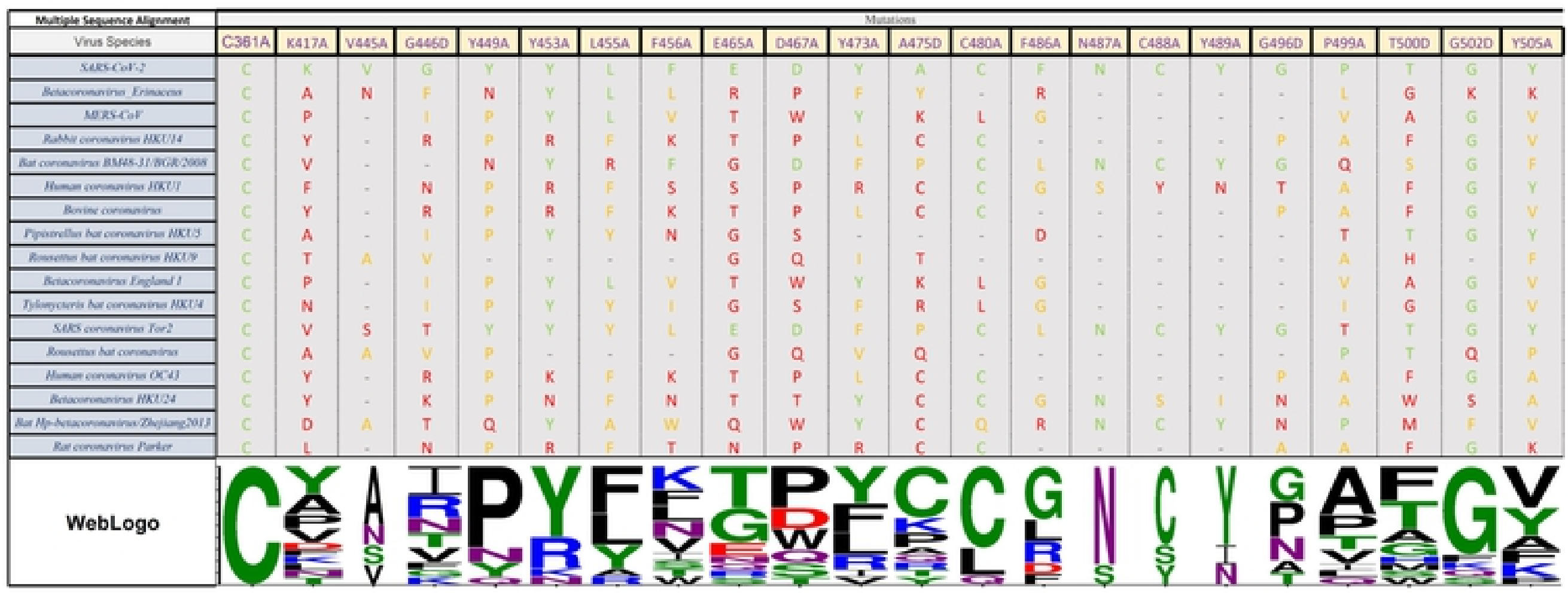

bioRxiv preprint doi: https://doi.org/10.1101/2021.01.29.428773; this version posted January 29, 2021. The copyright holder for this preprint (which was not certified by peer review) is the author/funder, who has granted bioRxiv a license to display the preprint in perpetuity. It is made available under aCC-BY 4.0 International license.

B

SARS-CoV-2

Pangolin-CoV

Bat-CoV-RaTG13

SARS-CoV-2

Pangolin- $\mathrm{CoV}$

Bat-CoV-RaTG13
C361A. K417A .V445A. G446D. Y449A. Y453A. L455A . F456A

NRKR I S N C VA D Y PG QT GK| I A DYN Y K I AWN S N N L D S KV|G G N Y N Y L Y R L L F R S N NRKR I S N C VADYPGQT GR I ADYNYKI AWN SNNLD S V G G NYN Y L Y R L F R K S N

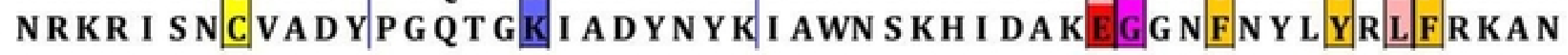

E465A . D467A . Y473A . A475D . C480A . F486A . N487A . C488A . Y489A . G496D . P499A . T500D . G502D . Y505A K P F ERDI S T E I Y QA A S T P C N G VE GF N C Y F P L Q S Y GF Q P T NG V G Y Q P Y R VVVL K P F E R D I S T E I Y Q A G S T P C N G VEG F N C Y F P L Q S Y G F H P T N G V G Y Q P Y R VVVL

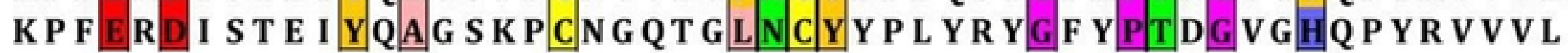

